Improved elimination of motion artifacts from a photoplethysmographic signal using a Kalman smoother with simultaneous accelerometry

This article has been downloaded from IOPscience. Please scroll down to see the full text article.

2010 Physiol. Meas. 311585

(http://iopscience.iop.org/0967-3334/31/12/003)

View the table of contents for this issue, or go to the journal homepage for more

Download details:

IP Address: 147.46.182.184

The article was downloaded on 25/05/2011 at 07:00

Please note that terms and conditions apply. 


\title{
Improved elimination of motion artifacts from a photoplethysmographic signal using a Kalman smoother with simultaneous accelerometry
}

\author{
Boreom Lee ${ }^{1}$, Jonghee Han ${ }^{2}$, Hyun Jae Baek ${ }^{3}$, Jae Hyuk Shin ${ }^{4}$, \\ Kwang Suk Park ${ }^{5}$ and Won Jin Yi ${ }^{6,7}$ \\ ${ }^{1}$ Graduate-Program of Medical System Engineering (GMSE), Gwangju Institute of Science and \\ Technology (GIST), Gwangju, Korea \\ 2 Department of Biomedical Engineering, Hanyang University, Seoul, Korea \\ ${ }^{3}$ Graduate Program in Bioengineering, Seoul National University, Seoul, Korea \\ ${ }^{4}$ Interdisciplinary program of Biomedical Engineering, Seoul National University, Seoul, Korea \\ ${ }^{5}$ Department of Biomedical Engineering, College of Medicine, Seoul National University, Seoul, \\ Korea \\ ${ }^{6}$ Department of Oral and Maxillofacial Radiology, BK21, and Dental Research Institute, School \\ of Dentistry, Seoul National University, Seoul, Korea \\ E-mail: leebr@gist.ac.kr, apaper@bme.hanyang.ac.kr, hyunjae100@bmsil.snu.ac.kr, \\ russell@bmsil.snu.ac.kr, kspark@bmsil.snu.ac.kr and wjyi@snu.ac.kr
}

Received 4 June 2010, accepted for publication 1 October 2010

Published 27 October 2010

Online at stacks.iop.org/PM/31/1585

\begin{abstract}
A photoplethysmography (PPG) signal provides very useful information about a subject's hemodynamic status in a hospital or ubiquitous environment. However, PPG is very vulnerable to motion artifacts, which can significantly distort the information belonging to the PPG signal itself. Thus, the reduction of the effects of motion artifacts is an important issue when monitoring the cardiovascular system by PPG. There have been many adaptive techniques to reduce motion artifacts from PPG signals. In the present study, we compared a method based on the fixed-interval Kalman smoother with the usual adaptive filtering algorithms, e.g. the normalized least mean squares, recursive least squares and the conventional Kalman filter. We found that the fixed-interval Kalman smoother reduced motion artifacts from the PPG signal most effectively. Therefore, the use of the fixed-interval Kalman smoother can reduce motion artifacts in PPG, thus providing the most reliable information that can be deduced from the reconstructed PPG signals.
\end{abstract}

Keywords: photoplethysmography, motion artifact, fixed-interval Kalman smoother, Kalman filter, NLMS, RLS

(Some figures in this article are in colour only in the electronic version)

7 Author to whom any correspondence should be addressed. 


\section{Introduction}

A photoplethysmography (PPG) signal provides useful cardiovascular and respiratory information, like arterial oxygen saturation, heart rate and respiratory rate (Shelley 2007). Recently, the PPG signal was used instead of electrocardiography (ECG) to estimate heart rate variability (HRV) (Lu et al 2008). In addition, the PPG signal can be used to measure pulse arrival time (PAT), combined with ECG, to estimate blood pressure (Baek et al 2010). PPG recording is a very convenient tool for continuous and ubiquitous monitoring of the hemodynamic status of healthy individuals or patients. It can measure pulse signals noninvasively and can also be developed as a portable device in order to monitor physiologic condition during daily activities (Asada et al 2003).

However, the PPG signal is easily corrupted by motion artifact both in bed-side patientmonitoring and in continuous mobile monitoring using portable devices (Hayes and Smith 1998). Motion artifacts distort the information contained in the PPG signals, such that the removal of motion artifact is important for the exact estimation of physiological variables and assessment of cardiovascular status, including heart rate, HRV and oxygen saturation.

To date, a number of methods have been suggested for the removal of a motion artifact from PPG signals. Kim et al proposed independent component analysis exploiting the quasiperiodicity of PPG signals (Kim and Yoo 2006). In addition, wavelet-based methods (Foo 2006) or non-linear methods (Hayes and Smith 1998) have also been suggested. There have also been many attempts to utilize adaptive filtering techniques for the on-line reduction of motion artifacts. Foo (2006) used the least mean squares (LMS) iterative algorithm and Gibbs et al (2005) used the recursive least-squares (RLS) adaptive noise cancellation technique to obtain clean filtered PPG signals from those corrupted by motion artifacts. In addition, it was reported that the normalized least mean squares (NLMS) algorithm (Han et al 2007) and the adaptive formula based on the Kalman filter (Seyedtabaii and Seyedtabaii 2008) successfully reduced the motion artifacts from the corrupted PPG signals. Similarly, adaptive filtering based on the Kalman formulation was used in ballistocardiogram artifact removal from electroencephalographies (EEGs) acquired during magnetic resonance image (MRI) scanning (In et al 2006, Bonmassar et al 2002).

In this study, we propose a fixed-interval Kalman smoother technique for the purpose of improving the elimination of motion artifact from PPG signals. We compare our proposed method with other adaptive filtering techniques, such as the NLMS filter, RLS filter and conventional Kalman filter, by applying the algorithms to simulations and to real experimental data. The organization of this paper is as follows. In section 2, the algorithms to be compared are formulated to be suitable for the adaptive reduction scheme of motion artifacts. Next, section 3 shows the results from simulation and actual experiments processed by the algorithms presented in section 2. In the last section, we consider and discuss some points on the results for each of the presented methods as they were applied to simulation and experimental data.

\section{Algorithms}

In this section, the following algorithms were formulated according to the adaptive noise cancellation scheme: NLMS, RLS, conventional Kalman filter and fixed-lag Kalman smoother. Detailed comparisons of their performances in the elimination of motion artifacts from the PPG signal are described in later sections. 


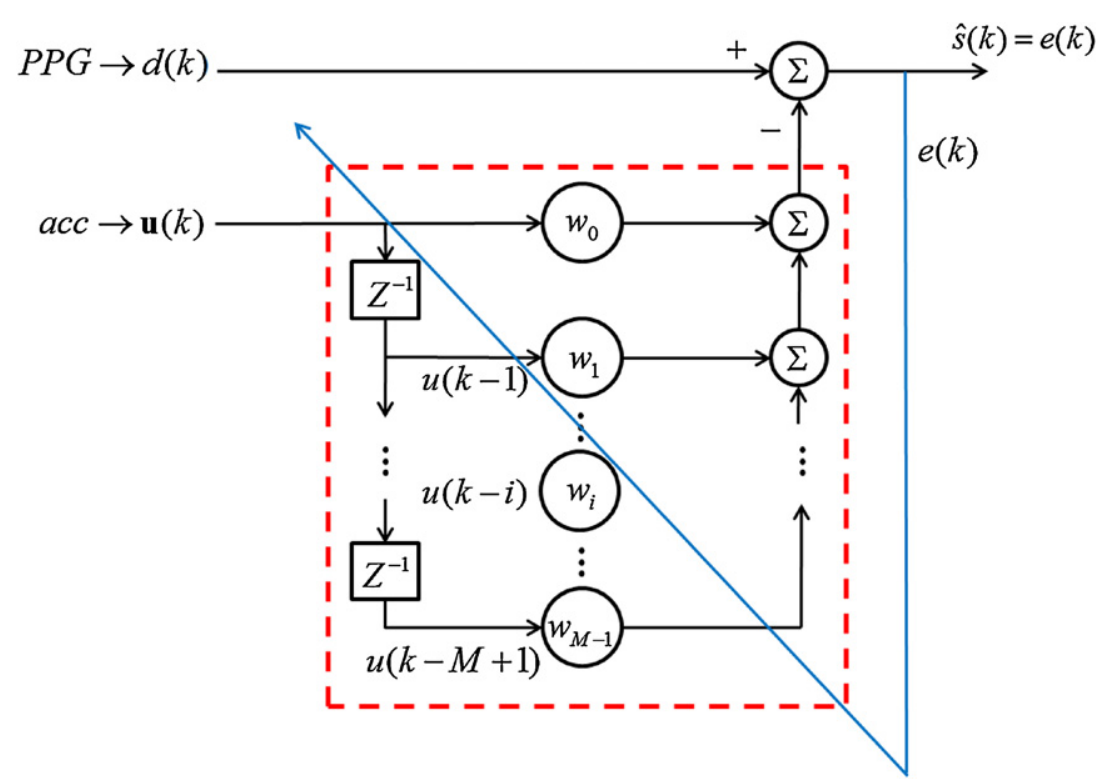

Figure 1. Dynamic structure of the adaptive FIR filter to eliminate motion artifacts from PPG signals, which can be represented by a multiple regression model (see (1), acc means accelerometer signal).

\subsection{Adaptive filters}

Adaptive recursive filters have been widely used for real-time removal of motion artifacts in many biomedical applications, including ECG and PPG signals, because the transfer function from motion to artifacts in a physiological system cannot be easily described by a definite physical model. In this study, we constructed several algorithms of adaptive noise cancellation having finite impulse response (FIR) structures. The structure of the system can be described by the multiple regression model, as in (1), and the basic idea is represented schematically in figure 1:

$$
d(k)=\mathbf{w}^{T} \mathbf{u}(k)+e(k)
$$

where $\mathbf{w}$ is an unknown parameter vector corresponding to filter tap coefficients with order $M$, and $d(k), e(k)$ and $\mathbf{u}(k)$ are the desired response, measurement error and filter tap input vector at time $k$, respectively (Haykin 2002). The superscript $T$ stands for the transpose of the matrix or vector. In the present study, $d(k)$ represents the measured PPG signals that are distorted by motion artifacts.

According to the specific procedures for the adaptation of filter tap weights, there are several algorithms, such as the LMS and RLS methods. For example, in the LMS algorithm, the filter weights are adapted according to the Widrow-Hoff learning rule, as follows:

$$
\hat{\mathbf{w}}(k+1)=\hat{\mathbf{w}}(k)+\mu \mathbf{u}(k) e(k)
$$

where $\hat{\mathbf{w}}(k)$ is the estimate of filter weights and $\mathbf{u}(k)$ is an input vector provided by an accelerometer signal at time $k$ and $e(k)$ is the estimation error at time $k$ obtained by

$$
e(k)=d(k)-\hat{\mathbf{w}}^{T}(k) \mathbf{u}(k)
$$

from which the PPG signal with reduced motion artifacts can be estimated, such as $\hat{s}(k)=e(k)$ (In et al 2006, Hayes 1996). 
The adjustment proportional to the tap-input vector $\mathbf{u}(k)$ causes a gradient noise amplification problem in the LMS method when $\mathbf{u}(k)$ is large. Therefore, by using normalization during the processes of tap-weight adaptation with the squared Euclidean norm of the tap-input vector $\mathbf{u}(k)$, the above-mentioned problem can be avoided. Thus, the algorithm is named as normalized LMS (NLMS) and the filter tap-weights can be adjusted according to

$\hat{\mathbf{w}}(k+1)=\hat{\mathbf{w}}(k)+\frac{\mu}{\|\mathbf{u}(k)\|^{2}+\delta} \mathbf{u}(k) e(k)=\hat{\mathbf{w}}(k)+\frac{\mu}{\mathbf{u}^{T}(k) \mathbf{u}(k)+\delta} \mathbf{u}(k) e(k)$

where $0<\mu<2$ and a small positive number $(\delta)$ is added in the denominator for normalization, to cope with the case of a $\|\mathbf{u}(k)\|$ that is too small (Hayes 1996). In this study, the NLMS algorithm was compared with other algorithms, such as the RLS and Kalman recursion-based methods in the later sections.

\subsection{Conventional Kalman filter}

The aforementioned multiple regression model can be described as the state-space formulation from which Kalman recursion algorithms can be derived. In addition, LMS and RLS adaptive filters can be generalized to the Kalman filter representations (Tarvainen et al 2004). Statespace representation can be expressed by the following equations (Simon 2006):

$$
\begin{aligned}
\mathbf{x}_{k} & =\mathbf{A}_{k-1} \mathbf{x}_{k-1}+\mathbf{n}_{k-1} \\
y_{k} & =\mathbf{C}_{k} \mathbf{x}_{k}+e_{k},
\end{aligned}
$$

in which (5) is a state equation and (6) is an observation equation. $\mathbf{x}_{k}$ is the state vector of the system, $\mathbf{A}_{k}$ is the transition matrix, $\mathbf{C}_{k}$ is the observation matrix and $y_{k}$ is the observation data. $\mathbf{n}_{k}$ is the system noise vector and $e_{k}$ is the observation noise, which are both assumed to be zeromean white Gaussian noise processes, respectively, e.g. $\mathbf{n}_{k} \sim N\left(\mathbf{0}, \mathbf{Q}_{k}\right)$ and $e_{k} \sim N\left(0, \sigma_{k}^{2}\right)$, and to be independent of each other. $\mathbf{Q}_{k}$ is the state noise covariance matrix and $\sigma_{k}^{2}$ is the measurement noise variance. In this study, the state vector can be expressed as the random walk model, which makes the state transition matrix $\mathbf{A}_{k}$ the identity matrix:

$$
\mathbf{x}_{k}=\mathbf{x}_{k-1}+\mathbf{n}_{k-1} \text {. }
$$

From (5') and (6), the following Kalman recursion equations can be obtained as (Simon 2006)

$$
\begin{aligned}
\hat{\mathbf{x}}_{k}^{-} & =\hat{\mathbf{x}}_{k-1}^{+} \\
\mathbf{P}_{k}^{-} & =\mathbf{P}_{k-1}^{+}+\mathbf{Q}_{k} \\
\mathbf{K}_{k} & =\mathbf{P}_{k}^{-} \mathbf{C}_{k}^{T}\left(\mathbf{C}_{k} \mathbf{P}_{k}^{-} \mathbf{C}_{k}^{T}+\mathbf{R}_{k}\right)^{-1} \\
\hat{\mathbf{x}}_{k}^{+} & =\hat{\mathbf{x}}_{k}^{-}+\mathbf{K}_{k}\left(y_{k}-\mathbf{C}_{k} \hat{\mathbf{x}}_{k}^{-}\right) \\
\mathbf{P}_{k}^{+} & =\left(\mathbf{I}-\mathbf{K}_{k} \mathbf{C}_{k}\right) \mathbf{P}_{k}^{-}
\end{aligned}
$$

where $\mathbf{K}_{k}$ is called the Kalman filter gain, which updates the state estimate when a new observation is detected, $\hat{\mathbf{x}}_{k}^{-}$and $\mathbf{P}_{k}^{-}$are the state vector estimate and its covariance matrix before $y_{k}$ is observed, and $\hat{\mathbf{x}}_{k}^{+}$and $\mathbf{P}_{k}^{+}$are the state vector estimate and its covariance matrix after $y_{k}$ is observed.

If considering the previous scheme of the adaptive filter, $\hat{\mathbf{x}}_{k}^{+}$and $\hat{\mathbf{x}}_{k}^{-}$correspond to the tap-weight estimates, $\hat{\mathbf{w}}(k)$ and $\hat{\mathbf{w}}(k-1)$ respectively, $y_{k}$ becomes the desired response $d(k)$, and tap-input $\mathbf{u}^{T}(k)$ can replace $\mathbf{C}_{k}$. With additional replacement of $\mathbf{P}_{k}^{-}$by $\mathbf{P}_{k-1}$, the recursive update equations can be expressed as 
Table 1. The relationship between the recursive algorithms (KF, RLS and NLMS) with respect to state-space perspective. $\mathbf{K}_{k}$ is the Kalman gain vector and $\mathbf{P}_{k}$ is the conditional covariance matrices of the parameter estimation error.

\begin{tabular}{lll}
\hline & $\mathbf{K}_{k}$ & $\mathbf{P}_{k}$ \\
\hline $\mathrm{KF}$ & $\mathbf{P}_{k-1} \mathbf{C}_{k}^{T}\left(\mathbf{C}_{k} \mathbf{P}_{k-1} \mathbf{C}_{k}^{T}+\mathbf{R}_{k}\right)^{-1}$ & $\left(\mathbf{I}-\mathbf{K}_{k} \mathbf{C}_{k}\right) \mathbf{P}_{k-1}+\mathbf{Q}_{k}$ \\
RLS & $\mathbf{P}_{k-1} \mathbf{C}_{k}^{T}\left(\mathbf{C}_{k} \mathbf{P}_{k-1} \mathbf{C}_{k}^{T}+\lambda\right)^{-1}$ & $\lambda^{-1}\left(\mathbf{I}-\mathbf{K}_{k} \mathbf{C}_{k}\right) \mathbf{P}_{k-1}$ \\
NLMS $^{\mathrm{a}}$ & $\mu_{1} \mu_{2} \mathbf{C}_{k}^{T}\left(\mu_{2} \mathbf{C}_{k} \mathbf{C}_{k}^{T}+1\right)^{-1}$ & $\mu_{2} \mathbf{I}$ \\
\hline
\end{tabular}

$\mathrm{KF}=$ Kalman filter; $\mathrm{RLS}=$ recursive least-squares method; NLMS $=$ normalized least mean squares method.

${ }^{a}$ Considering (4), $\mu_{1}$ corresponds to $\mu$ and $\mu_{2}$ corresponds to $1 / \delta$.

$$
\begin{aligned}
& \mathbf{K}_{k}=\mathbf{P}_{k-1} \mathbf{u}(k) /\left(\mathbf{u}^{T}(k) \mathbf{P}_{k-1} \mathbf{u}(k)+\sigma_{k}^{2}\right) \\
& \hat{\mathbf{w}}(k)=\hat{\mathbf{w}}(k-1)+\mathbf{K}_{k}\left(d(k)-\mathbf{u}^{T}(k) \hat{\mathbf{w}}(k-1)\right) \\
& \mathbf{P}_{k}=\left(\mathbf{I}-\mathbf{K}_{k} \mathbf{u}^{T}(k)\right) \mathbf{P}_{k-1}+\mathbf{Q}_{k},
\end{aligned}
$$

which can also represent the state-space formulation of the RLS adaptive filter if $\sigma_{k}^{2}$ is replaced by $\lambda(0.9 \leqslant \lambda \leqslant 1)$ in $\left(9^{\prime}\right)$, along with $\mathbf{P}_{k}=\lambda^{-1}\left(\mathbf{I}-\mathbf{K}_{k} \mathbf{C}_{k}\right) \mathbf{P}_{k-1}\left(\mathbf{Q}_{k}=\mathbf{0}\right)$ replaced in (11') (Tarvainen et al 2004, Haykin 2002). The formulations of the algorithms (NLMS, RLS and Kalman filter) can be compared with respect to state-space perspective, and the relationships between them are summarized in table 1 (Georgiadis et al 2005).

Usually, the state noise covariance matrix $\left(\mathbf{Q}_{k}\right)$ can be simplified as $\mathbf{Q}_{k}=\sigma_{\mathrm{kf}}^{2} \mathbf{I}$ and the measurement noise variance $\left(\sigma_{k}^{2}\right)$ can be set to $1\left(\sigma_{k}^{2}=\sigma^{2}=1\right)$ since it is known that only the ratio $\sigma^{2} / \sigma_{\mathrm{kf}}^{2}$ influences the estimates (Tarvainen et al 2006, Georgiadis et al 2005). Additionally, the optimal parameters of algorithms were selected specific to each data to obtain the best estimates ( $\mu_{\max }$ for NLMS, $\lambda_{\max }$ for RLS and $\sigma_{\mathrm{kf} \text {, max }}^{2}$ for the Kalman filter) (Tarvainen et al 2004).

\subsection{Fixed-interval Kalman smoother}

If an immediate update of the state estimate is not necessary and we can have a little time lag from the time points of observations, the future observations can be used for the estimation of the state. Such estimators are called smoothers. If we have observations for a fixed time interval (after getting all the data points), the state estimate at the interior time point $k(k=1, \ldots, T)$ of the time interval can be obtained given the observations $y_{1}, \ldots, y_{T}$ and the estimator is called the fixed-interval smoother (Tarvainen et al 2004). In this study, we implemented the RTS (Rauch-Tung-Striebel) smoother, which is more difficult to understand but is computationally more efficient than the forward-backward approach (Simon 2006).

In the implementation of the fixed-interval Kalman smoother, the standard forward Kalman filter must be executed initially and, thereafter, the backward RTS smoothing can be processed from $k=T$ to $k=1$ iteratively. The additional procedures are as follows:

$$
\begin{aligned}
& \mathbf{K}_{\mathbf{k}}^{\prime}=\mathbf{P}_{k}^{+}\left(\mathbf{P}_{k+1}^{-}\right)^{-1} \\
& \hat{\mathbf{x}}_{k}^{\prime}=\hat{\mathbf{x}}_{k}^{+}+\mathbf{K}_{\mathbf{k}}^{\prime}\left(\hat{\mathbf{x}}_{k+1}^{\prime}-\hat{\mathbf{x}}_{k+1}^{-}\right)
\end{aligned}
$$

where the smoother gain matrix $\mathbf{K}_{\mathbf{k}}^{\prime}$ of the RTS method includes the error covariances obtained during the forward processes, and the RTS estimate $\hat{\mathbf{x}}_{k}^{\prime}$ also requires the state estimates resulting from the forward Kalman filtering, including the initial condition of $\hat{\mathbf{x}}_{T}^{\prime}=\hat{\mathbf{x}}_{T}^{+}$. Like other 
algorithms, the optimal parameters were selected for each data to obtain the best estimates $\left(\sigma_{\mathrm{ks}, \max }^{2}\right.$ for the Kalman smoother) (Tarvainen et al 2004). To follow the above dynamic scheme of adaptive filtering, (12) and (13) can be reformulated as follows:

$$
\begin{aligned}
& \mathbf{K}_{\mathbf{k}}^{\prime}=\mathbf{P}_{k}^{+}\left(\mathbf{P}_{k}\right)^{-1} \\
& \hat{\mathbf{w}}^{\prime}(k)=\hat{\mathbf{w}}(k)+\mathbf{K}_{\mathbf{k}}^{\prime}\left(\hat{\mathbf{w}}^{\prime}(k+1)-\hat{\mathbf{w}}(k+1)\right)
\end{aligned}
$$

where $\mathbf{P}_{k}$ corresponds to $\mathbf{P}_{k+1}^{-}, \hat{\mathbf{w}}(k+1)$ corresponds to $\hat{\mathbf{x}}_{k+1}^{-}$and $\mathbf{P}_{k}^{+}$must be stored in advance during the forward Kalman process as well as $\mathbf{P}_{k}$ and $\hat{\mathbf{w}}(k)$.

\subsection{Relationship between algorithms}

The Kalman filter provides an optimal estimate in the mean-square sense and can be formulated as a state-space model. RLS can also be described by a state-space model (Chun et al 1998) and can be recognized as a special case of the Kalman filter (Sayed 2008, Sayed and Kailath 1994). The RLS method and Kalman filter showed comparable performances in several applications (Barnawi et al 2007, Chun et al 1998, Lanzi et al 2006). In addition, RLS is known to be more robust and to converge faster than NLMS (Haykin 2002). On the other hand, optimal smoothers estimate the state of a system at a given time point using more measurements made before and after that time point and therefore the accuracy of a smoother is generally superior to that of a filter. Optimal smoothers can be derived from the Kalman filter formulation (Grewal and Andrews 2008). In addition, using the Kalman smoother can avoid the lag error present in adaptive filters such as the Kalman filter, RLS or NLMS (Tarvainen et al 2006). From the point of computation and memory demand, NLMS has an order of complexity $O(M)$ per iteration while RLS and Kalman filter have an order of $O\left(M^{2}\right)$ per iteration (Lanzi et al 2006, Sayed and Kailath 1994). A fixed-interval Kalman smoother is computationally heaviest among the algorithms, needs a large memory capacity and can only be used in off-line processing unlike others (Grewal and Andrews 2008).

\section{Simulations and real experiments}

In this section, the algorithms will be applied to the simulation data and then the real experimental data. The performance results for the algorithms were compared with each other. Tests for the algorithms were performed in the MATLAB (MathWorks Inc., USA) environment.

\subsection{Simulations}

3.1.1. Preparation of simulated PPG signals. To construct the simulation dataset, the motion artifacts were modeled by sine functions with 1,2 or $3 \mathrm{~Hz}$ because the finger and hand motions in daily activity are low-frequency movements (Kunesch et al 1989, Xiong and Quek 2006). These artificial signals of motion artifact and white Gaussian noise were merged with the real PPG signals, which were obtained at resting state using a commercial PPG sensor and customized acquisition device. We prepared two simulation datasets using $1 \mathrm{~Hz}$ and $2 \mathrm{~Hz}$ resting PPG signals together with different SNRs (signal-to-noise ratios) and tested each algorithm using these same datasets with six different filter tap orders. The first dataset (simulation 1) was constructed by merging a resting PPG having about $2 \mathrm{~Hz}$ (about $120 \mathrm{bpm}$ ) cycle and $45 \mathrm{~s}$ duration (see figure 2(a)) with artificial motion artifacts made from $1 \mathrm{~Hz}, 2 \mathrm{~Hz}$, $3 \mathrm{~Hz}$ sinusoids, as well as mixed sinusoids with 1 and $2 \mathrm{~Hz}$, with 1 and $3 \mathrm{~Hz}$, and with 2 and $3 \mathrm{~Hz}$ components. The second dataset (simulation 2) was constructed by merging a resting PPG having about $1 \mathrm{~Hz}$ (about $60 \mathrm{bpm}$ ) cycle and $66 \mathrm{~s}$ duration (see figure 2(b)) with artificial 
motion artifacts made from $1 \mathrm{~Hz}, 2 \mathrm{~Hz}, 3 \mathrm{~Hz}$ sinusoids, as well as mixed sinusoids with 1 and $2 \mathrm{~Hz}$, with 1 and $3 \mathrm{~Hz}$, and with 2 and $3 \mathrm{~Hz}$ components. In addition, the artificial motion artifacts were mingled to the real PPG signal with different SNRs of $-12.5,-10,-7.5,-5$ and $-2.5 \mathrm{~dB}$. One example of an artificial PPG signal mixed with a $2 \mathrm{~Hz}$ motion artifact (MA) having a SNR of $-7.5 \mathrm{~dB}$ of simulation dataset 1 is displayed in figure 2(a). In figure 2(a), the uppermost plot of the left column overlays the original real PPG signal (dotted line) and artificially motion artifact-corrupted PPG signal (thin solid line), and the lower plots of the left column represent the signals reconstructed by each algorithm (thick solid lines), i.e. NLMS, RLS, Kalman filter and fixed-interval Kalman smoother, which are overlaid over the original and simulated PPG signals. In the right column of figure 2(a), the selection procedures for the optimal parameters of each method are displayed, such that the reconstructed signals represent the maximal cross correlation with the original PPG signal. Another example of the artificial PPG signal mixed with $1 \mathrm{~Hz}$ MA having a SNR of $-5 \mathrm{~dB}$ of simulation dataset 2 is displayed in figure 2(b) in a similar way to figure 1(a).

3.1.2. Results of simulation data. Four algorithms were applied to the two simulation datasets, which respectively comprise six artificial motion artifacts $(1 \mathrm{~Hz}, 2 \mathrm{~Hz}, 3 \mathrm{~Hz}, 1$ and $2 \mathrm{~Hz}, 1$ and $3 \mathrm{~Hz}$, and 2 and $3 \mathrm{~Hz}$ MA) with five different SNR levels (-12.5 - 2.5 dB). All results are summarized in figures 3 (simulation dataset 1) and 4 (simulation dataset 2) for all of the combinations of datasets with six different FIR filter tap orders $(8,16,24,32,40$ and 48 ), and, for each situation, the optimal parameters of each algorithm ( $\mu_{\max }$ for NLMS, $\lambda_{\max }$ for RLS, $\sigma_{\mathrm{kf}, \max }^{2}$ for the Kalman filter, and $\sigma_{\mathrm{ks} \text {, max }}^{2}$ for the Kalman smoother) were searched to represent the results showing the maximal values of cross correlations between the original PPG signal and the artifact-rejected PPG signals. Four representative examples of simulation dataset 1 and four of simulation dataset 2 are arranged in table 2, which gives the specific numerical values for the cross correlations between the original PPG signal and the simulated signals of PPG with motion artifacts, and between the original signal and reconstructed PPG signals acquired after processing by four algorithms, respectively. In addition, table 2 shows the optimal values of parameters for each method in order to obtain the maximal cross correlation between the reconstructed PPG signals and the original PPG signal.

For all the combinations of the two datasets with different SNR levels and different filter tap orders, the results of the fixed-interval Kalman smoother represented PPG signals which were most correlated with the original PPG signal. The results of a conventional Kalman filter and RLS generally showed similar results, but the Kalman filter represented a more stable and accurate reconstruction than RLS because the results of the RLS filter showed much lower values than those for the Kalman filter in several cases of simulation data (the cases of 1 and $2 \mathrm{~Hz}$ MA with $-12.5,-10$ and $-7.5 \mathrm{dBs}$ in simulation 1 and all cases of 1 and $2 \mathrm{~Hz} \mathrm{MA}$ in simulation 2 above the tap order of 24, see figures 3 and 4). According to our simulation results, the NLMS method showed the lowest levels of cross correlation between the filtered PPG signals and the original PPG signal among the algorithms in most cases (but not in all the cases). As SNR increases, the results of the Kalman filter, RLS and NLMS tended to converge each other. However, the fixed-interval Kalman smoother showed the best results out of the four algorithms in all the simulated PPG signals and filter tap orders to the limit of our simulation tests.

\subsection{Experiments}

3.2.1. Acquisition of real PPG signals. The PPG and accelerometer signals, which were measured in a real situation, were processed by NLMS, RLS, Kalman filter and fixed-interval 


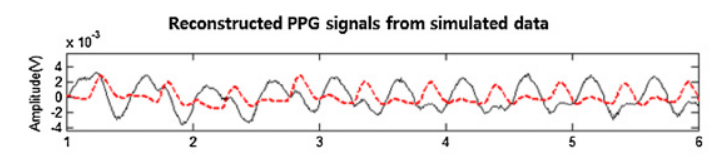

年
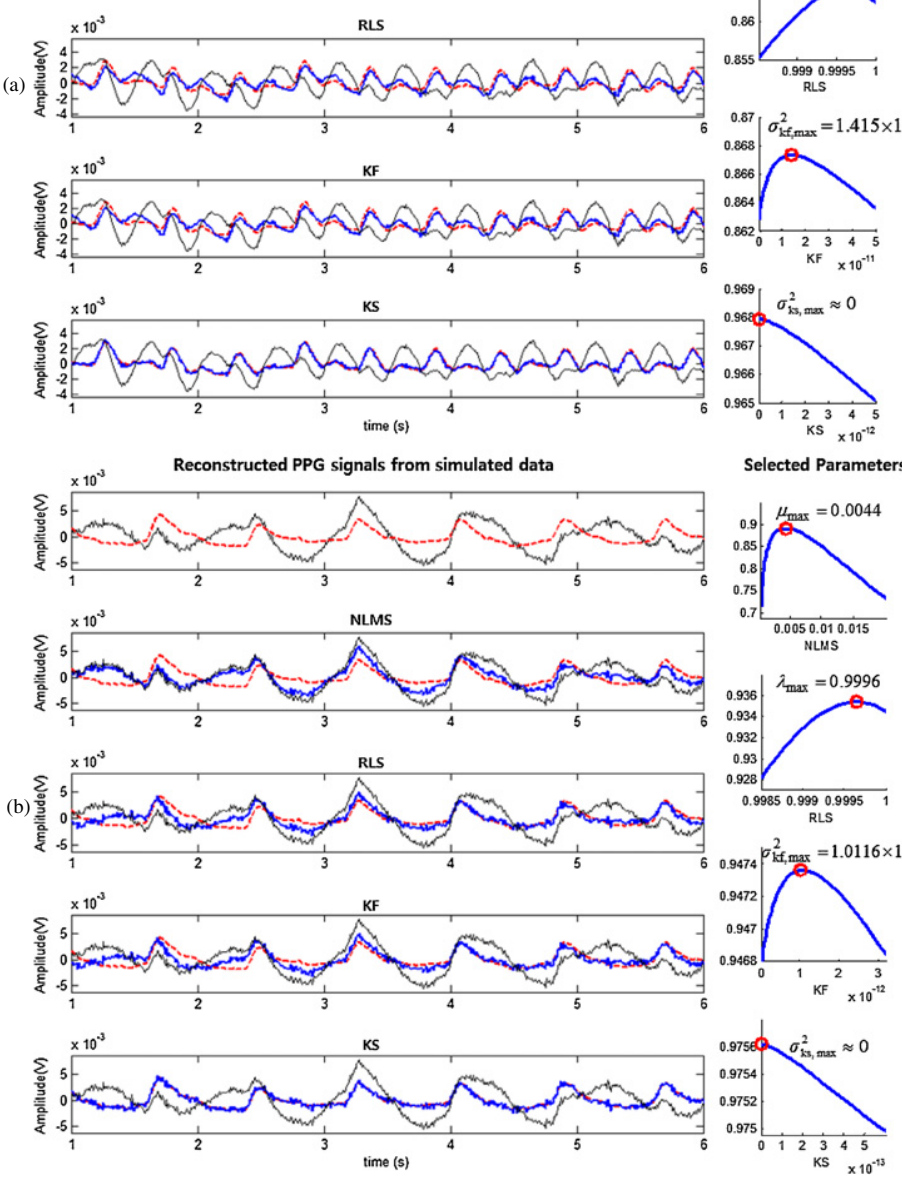

Figure 2. (a) One example of simulation dataset 1 is displayed. The original PPG (nearly $2 \mathrm{~Hz}$ cycle) measured at rest was mixed with artificial motion artifact having $2 \mathrm{~Hz}$ fluctuation, which represents an SNR of $-7.5 \mathrm{~dB}$. The adaptive FIR filter tap order is 16 in this sample analysis and results are displayed from 1 to $6 \mathrm{~s}$. The uppermost plot of the left column overlays the original real PPG signal (dotted line) and artificially motion artifact-corrupted PPG signal (thin solid line) and the lower plots represent the signals reconstructed by each algorithms (thick solid lines), i.e. NLMS, RLS, Kalman filter, and fixed-interval Kalman smoother, which are overlaid over the original and simulated PPG signals. The cross correlation was 0.2567 between the original PPG and the PPG with artificial motion artifact before filtering. As in plots of the right column, optimal parameters for each method are selected, such that the reconstructed signals represent the maximal cross correlation with the original PPG signal respectively ( $x$-axis: parameter for each algorithm, $y$-axis: cross correlation). (b) Another example of simulation dataset 2 is displayed. The original PPG (nearly $1 \mathrm{~Hz}$ cycle) measured at rest was mixed with artificial motion artifact having $1 \mathrm{~Hz}$ fluctuation, which represents an SNR of $-5 \mathrm{~dB}$. The FIR filter tap order is 40 in this sample. The cross correlation was 0.4855 between the original PPG and the PPG with artificial motion artifact before filtering. Other explanations are the same as (a) SNR = signal-to-noise ratio. 

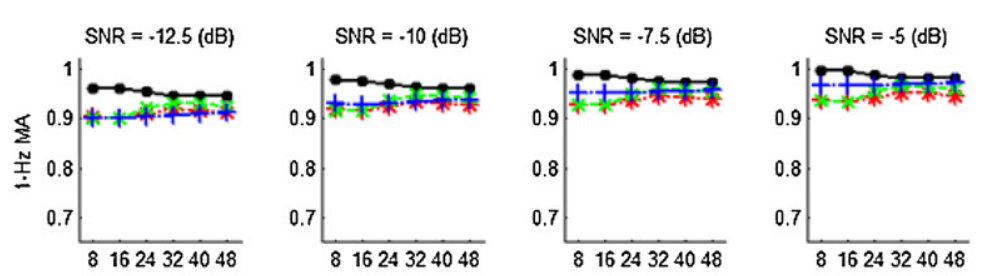

SNR $=-2.5(\mathrm{~dB})$
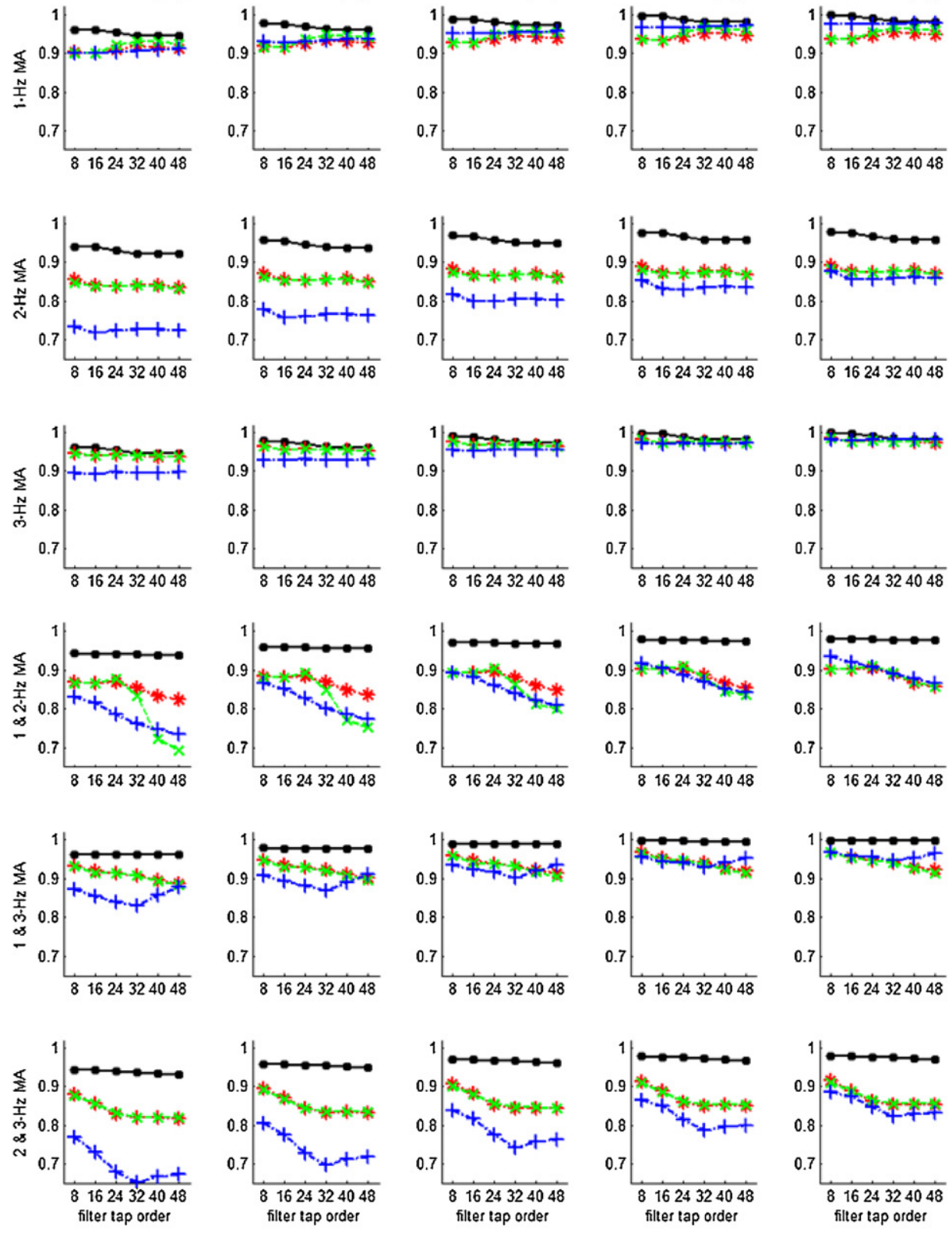

KS

$$
\text { ***.**. } \mathrm{KF}
$$

$$
-x-x-*-x-\text { RLS }
$$

$$
+\cdot+\cdot \text { NLMS }
$$

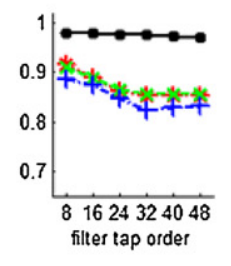

Figure 3. Four algorithms were applied to the simulation dataset 1 which comprise six different MAs with five different SNR levels $(-12.5$ to $-2.5 \mathrm{~dB})$. The maximal cross correlations were acquired for all the combinations of datasets with six different FIR filter tap orders $(8,16,24,32$, 40 and 48). For all the situations, the maximal cross correlations as well as the corresponding optimal parameters were searched with regard to each algorithm and the values are displayed with the $y$-axis in each plot. $Y$-axes represent the values of cross correlation between the filtered PPG signal and the original PPG signal. $\mathrm{KS}=$ fixed-interval Kalman smoother, $\mathrm{KF}=$ conventional Kalman filter, RLS $=$ recursive least-squares method, NLMS $=$ normalized least mean squares method, SNR = signal-to-noise ratio, $\mathrm{MA}=$ motion artifact. 

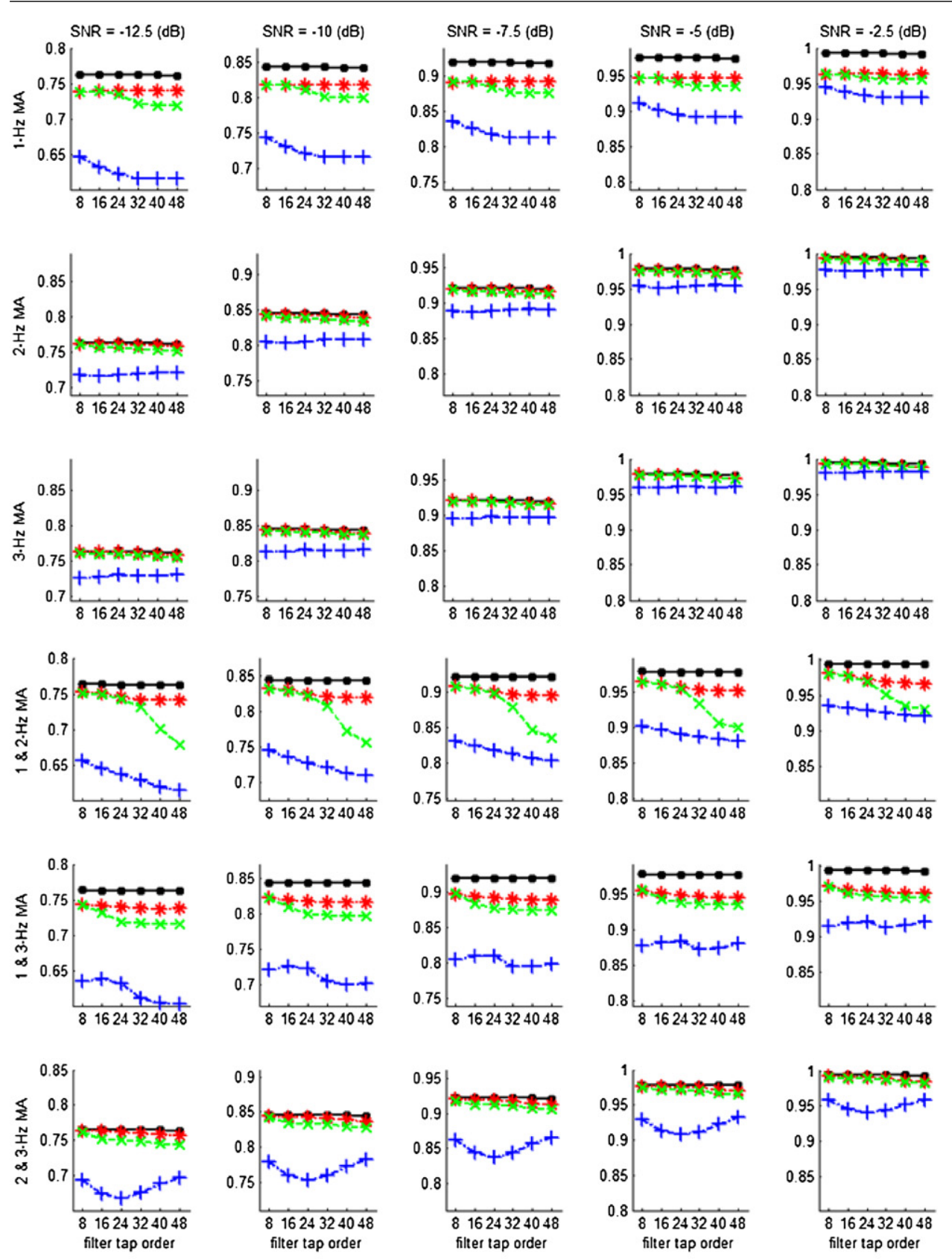

KS

***..**. KF

$-x--x-*-\operatorname{RLS}$

$+\cdot+\cdot+$ NLMS

Figure 4. Four algorithms were applied to the simulation dataset 2 which comprise six different MAs with five different SNR levels $(-12.5$ to $-2.5 \mathrm{~dB})$. The maximal cross correlations were acquired for all the combinations of datasets with six different FIR filter tap orders $(8,16,24,32$, 40 and 48). For all the situations, the maximal cross correlations as well as the corresponding optimal parameters were searched with regard to each algorithm and the values are displayed with the $y$-axis in each plot. $Y$-axes represent the values of cross correlation between the filtered PPG signal and the original PPG signal. $\mathrm{KS}=$ fixed-interval Kalman smoother, $\mathrm{KF}=$ conventional Kalman filter, RLS $=$ recursive least-squares method, NLMS $=$ normalized least mean squares method, SNR = signal-to-noise ratio, MA = motion artifact. 
Table 2. Cross correlations between the original PPG signal and the reconstructed PPG signals by four algorithms with regard to eight representative simulation data. CC correction between the original PPG signal and the simulated PPG distorted by artificial motion artifacts, $\mathrm{CC}_{\mathrm{KS}}$ is the cross correlation between the original PPG signal and the PPG reconstructed by fixed-interval Kalman smoother, $\mathrm{CC}_{\mathrm{KF}}$ is the cross correlation between the original PPG signal and the PPG reconstructed by the conventional Kalman filter, $\mathrm{CC}_{\mathrm{RLS}}$ is

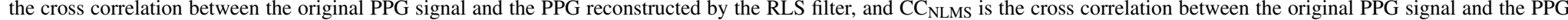
reconstructed by the NLMS filter. Values of optimal parameters for each algorithm are listed in the parentheses next to the values of cross correlation. MA $=$ motion artifact, SNR $=$ signal-to-noise ratio.

\begin{tabular}{|c|c|c|c|c|c|c|c|}
\hline \multirow[b]{2}{*}{ Dataset } & \multirow[b]{2}{*}{ Cross correlations } & \multicolumn{6}{|c|}{ Filter tap order } \\
\hline & & 8 & 16 & 24 & 32 & 40 & 48 \\
\hline Simulation 1 & $\mathrm{CC}_{\mathrm{KS}}\left(\sigma_{\mathrm{ks}, \max }^{2}\right)$ & $0.9693(\approx 0)$ & $0.9679(\approx 0)$ & $0.9594(\approx 0)$ & $0.9513(\approx 0)$ & $0.9503(\approx 0)$ & $0.9502(\approx 0)$ \\
\hline (2 Hz MA, & 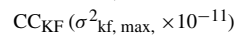 & $0.8818(3.2551)$ & $0.8673(1.4150)$ & $0.8643(1.2316)$ & $0.8692(1.0719)$ & $0.8697(0.9329)$ & $0.8612(0.7067)$ \\
\hline $\mathrm{SNR}=-7.5 \mathrm{~dB}$ & $\mathrm{CC}_{\mathrm{RLS}}\left(\lambda_{\max }\right)$ & $0.8740(0.9997)$ & $0.8643(0.9996)$ & $0.8644(0.9994)$ & $0.8678(0.9993)$ & $0.8670(0.9993)$ & $0.8597(0.9993)$ \\
\hline $\left.\mathrm{CC}_{\mathrm{MA}}=0.2567\right)$ & $\mathrm{CC}_{\mathrm{NLMS}}\left(\mu_{\max }\right)$ & $0.8184(0.0080)$ & $0.7974(0.0102)$ & $0.7977(0.0105)$ & $0.8034(0.0095)$ & $0.8048(0.0089)$ & $0.8016(0.0094)$ \\
\hline Simulation 1 & $\mathrm{CC}_{\mathrm{KS}}\left(\sigma^{2} \mathrm{ks}, \max \right)$ & $0.9593(\approx 0)$ & $0.9579(\approx 0)$ & $0.9578(\approx 0)$ & $0.9562(\approx 0)$ & $0.9546(\approx 0)$ & $0.9545(\approx 0)$ \\
\hline ( 1 and $2 \mathrm{~Hz} \mathrm{MA}$, & $\mathrm{CC}_{\mathrm{KF}}\left(\sigma_{\mathrm{kf}, \max ,}^{2} \times 10^{-12}\right)$ & $0.8831(\approx 0)$ & $0.8818(24.658)$ & 0.8844 (10.719) & 0.8694 (4.0555) & $0.8482(1.3355)$ & $0.8379(0.3331)$ \\
\hline $\mathrm{SNR}=-10 \mathrm{~dB}$ & $\mathrm{CC}_{\mathrm{RLS}}\left(\lambda_{\max }\right)$ & $0.8824(1)$ & $0.8822(1)$ & $0.8931(1)$ & $0.8479(1)$ & $0.7708(1)$ & $0.7521(1)$ \\
\hline $\left.\mathrm{CC}_{\mathrm{MA}}=0.1901\right)$ & $\mathrm{CC}_{\mathrm{NLMS}}\left(\mu_{\max }\right)$ & $0.8650(0.0116)$ & $0.8526(0.0165)$ & $0.8268(0.0192)$ & $0.8019(0.0243)$ & $0.7872(0.0277)$ & $0.7749(0.0269)$ \\
\hline Simulation 1 & $\mathrm{CC}_{\mathrm{KS}}\left(\sigma^{2} \mathrm{ks,max}, \times 10^{-13}\right)$ & $0.9787(1.9116)$ & $0.9773(\approx 0)$ & $0.9772(\approx 0)$ & $0.9755(\approx 0)$ & $0.9740(\approx 0)$ & $0.9738(\approx 0)$ \\
\hline ( 1 and $2 \mathrm{~Hz} \mathrm{MA}$, & $\mathrm{CC}_{\mathrm{KF}}\left(\sigma^{2}{ }_{\mathrm{kf}, \max ,} \times 10^{-11}\right)$ & $0.9010(\approx 0)$ & 0.9009 (11..357) & $0.9026(4.2970)$ & $0.8871(1.6258)$ & $0.8658(0.7067)$ & $0.8553(0.9329)$ \\
\hline $\mathrm{SNR}=-5 \mathrm{~dB}$ & $\mathrm{CC}_{\mathrm{RLS}}\left(\lambda_{\max }\right)$ & $0.9007(1)$ & $0.9010(1)$ & $0.9106(1)$ & $0.8802(1)$ & $0.8468(1)$ & $0.8356(1)$ \\
\hline $\left.\mathrm{CC}_{\mathrm{MA}}=0.4042\right)$ & $\mathrm{CC}_{\mathrm{NLMS}}\left(\mu_{\max }\right)$ & $0.9161(0.0053)$ & $0.9055(0.0098)$ & $0.8884(0.0111)$ & $0.8681(0.0128)$ & $0.8529(0.0149)$ & $0.8410(0.0150)$ \\
\hline Simulation 1 & $\mathrm{CC}_{\mathrm{KS}}\left(\sigma^{2} \mathrm{ks, \operatorname {max }}\right)$ & $0.9705(\approx 0)$ & $0.9702(\approx 0)$ & $0.9681(\approx 0)$ & $0.9660(\approx 0)$ & $0.9637(\approx 0)$ & $0.9600(\approx 0)$ \\
\hline (2 and $3 \mathrm{~Hz} \mathrm{MA}$, & $\mathrm{CC}_{\mathrm{KF}}\left(\sigma^{2}{ }_{\mathrm{kf}, \max ,} \times 10^{-12}\right)$ & $0.9061(24.658)$ & 0.8819 (8.1198) & $0.8537(4.6595)$ & $0.8441(7.0672)$ & $0.8449(9.3293)$ & $0.8438(8.1198)$ \\
\hline $\mathrm{SNR}=-7.5 \mathrm{~dB}$ & $\mathrm{CC}_{\mathrm{RLS}}\left(\lambda_{\max }\right)$ & $0.9020(1)$ & $0.8802(1)$ & $0.8560(0.9996)$ & $0.8471(0.9993)$ & $0.8461(0.9993)$ & $0.8450(0.9994)$ \\
\hline $\left.\mathrm{CC}_{\mathrm{MA}}=0.2704\right)$ & $\mathrm{CC}_{\text {NLMS }}\left(\mu_{\max }\right)$ & $0.8373(0.0081)$ & $0.8159(0.0096)$ & $0.7740(0.0102)$ & $0.7428(0.0104)$ & $0.7553(0.0115)$ & $0.7621(0.0116)$ \\
\hline Simulation 2 & $\mathrm{CC}_{\mathrm{KS}}\left(\sigma^{2} \mathrm{ks, \operatorname {max }}\right)$ & $0.9197(\approx 0)$ & $0.9197(\approx 0)$ & $0.9197(\approx 0)$ & $0.9193(\approx 0)$ & $0.9187(\approx 0)$ & $0.9180(\approx 0)$ \\
\hline (1 Hz MA, & $\mathrm{CC}_{\mathrm{KF}}\left(\sigma^{2} \mathrm{kf}, \max , \times 10^{-13}\right)$ & $0.8913(13.355)$ & $0.8922(15.344)$ & 0.8921 (11.623) & $0.8920(7.6634)$ & $0.8919(4.3976)$ & $0.8925(2.1964)$ \\
\hline $\mathrm{SNR}=-7.5 \mathrm{~dB}$, & $\mathrm{CC}_{\mathrm{RLS}}\left(\lambda_{\max }\right)$ & $0.8917(0.9997)$ & $0.8919(0.9997)$ & $0.8850(0.9997)$ & $0.8778(0.9997)$ & $0.8764(0.9998)$ & $0.8770(0.9998)$ \\
\hline $\left.\mathrm{CC}_{\mathrm{MA}}=0.3809\right)$ & $\mathrm{CC}_{\mathrm{NLMS}}\left(\mu_{\max }\right)$ & $0.8363(0.0040)$ & $0.8259(0.0044)$ & $0.8180(0.0048)$ & $0.8138(0.0050)$ & $0.8128(0.0051)$ & $0.8138(0.0051)$ \\
\hline Simulation 2 & $\mathrm{CC}_{\mathrm{KS}}\left(\sigma_{\mathrm{ks}, \max }^{2}\right)$ & $0.8445(\approx 0)$ & $0.8440(\approx 0)$ & $0.8435(\approx 0)$ & $0.8434(\approx 0)$ & $0.8434(\approx 0)$ & $0.8435(\approx 0)$ \\
\hline ( 1 and $2 \mathrm{~Hz} \mathrm{MA}$, & $\mathrm{CC}_{\mathrm{KF}}\left(\sigma^{2} \mathrm{kf, \operatorname {max } ,} \times 10^{-14}\right)$ & $0.8320(\approx 0)$ & $0.8299(9.5477)$ & $0.8246(25.235)$ & 0.8208 (28.994) & $0.8197(25.235)$ & $0.8197(19.116)$ \\
\hline $\mathrm{SNR}=-10 \mathrm{~dB}$ & $\mathrm{CC}_{\mathrm{RLS}}\left(\lambda_{\max }\right)$ & $0.8316(0.9999)$ & $0.8293(0.9999)$ & $0.8226(0.9998)$ & $0.8078(0.9997)$ & $0.7734(0.9998)$ & $0.7568(0.9998)$ \\
\hline $\left.\mathrm{CC}_{\mathrm{MA}}=0.2997\right)$ & $\mathrm{CC}_{\text {NLMS }}\left(\mu_{\max }\right)$ & $0.7448(0.0044)$ & $0.7358(0.0059)$ & $0.7275(0.0079)$ & $0.7216(0.0092)$ & $0.7130(0.0102)$ & $0.7090(0.0109)$ \\
\hline Simulation 2 & $\mathrm{CC}_{\mathrm{KS}}\left(\sigma_{\mathrm{ks}, \max }^{2}\right)$ & $0.9779(\approx 0)$ & $0.9772(\approx 0)$ & $0.9766(\approx 0)$ & $0.9765(\approx 0)$ & $0.9765(\approx 0)$ & $0.9765(\approx 0)$ \\
\hline ( 1 and $2 \mathrm{~Hz} \mathrm{MA}$, & $\mathrm{CC}_{\mathrm{KF}}\left(\sigma_{\mathrm{kf}, \max ,} \times 10^{-13}\right)$ & $0.9644(5.0526)$ & $0.9611(20.255)$ & $0.9560(26.738)$ & $0.9523(26.738)$ & $0.9508(23.272)$ & $0.9505(20.255)$ \\
\hline $\mathrm{SNR}=-5 \mathrm{~dB}$ & $\mathrm{CC}_{\mathrm{RLS}}\left(\lambda_{\max }\right)$ & $0.9646(0.9998)$ & $0.9611(0.9998)$ & $0.9538(0.9997)$ & $0.9328(0.9996)$ & $0.9066(0.9996)$ & $0.8998(0.9996)$ \\
\hline $\left.\mathrm{CC}_{\mathrm{MA}}=0.4906\right)$ & $\mathrm{CC}_{\mathrm{NLMS}}\left(\mu_{\max }\right)$ & $0.9003(0.0030)$ & $0.8954(0.0040)$ & $0.8902(0.0054)$ & $0.8869(0.0067)$ & $0.8826(0.0075)$ & $0.8805(0.0083)$ \\
\hline Simulation 2 & $\mathrm{CC}_{\mathrm{KS}}\left(\sigma^{2} \mathrm{ks}\right.$ max $)$ & $0.9215(\approx 0)$ & $0.9216(\approx 0)$ & $0.9217(\approx 0)$ & $0.9217(\approx 0)$ & $0.9216(\approx 0)$ & $0.9209(\approx 0)$ \\
\hline (2 and $3 \mathrm{~Hz} \mathrm{MA}$, & $\mathrm{CC}_{\mathrm{KF}}\left(\sigma^{2}{ }_{\mathrm{kf}, \max }\right)$ & $0.9198(\approx 0)$ & $0.9193(\approx 0)$ & $0.9188(\approx 0)$ & $0.9173(\approx 0)$ & $0.9145(\approx 0)$ & $0.9126(\approx 0)$ \\
\hline $\mathrm{SNR}=-7.5 \mathrm{~dB}$ & $\mathrm{CC}_{\mathrm{RLS}}\left(\lambda_{\max }\right)$ & $0.9181(1)$ & $0.9129(1)$ & $0.9122(1)$ & $0.9106(1)$ & $0.9077(1)$ & 0.9056 (0.9999) \\
\hline $\left.\mathrm{CC}_{\mathrm{MA}}=0.3823\right)$ & $\mathrm{CC}_{\mathrm{NLMS}}\left(\mu_{\max }\right)$ & $0.8625(0.0049)$ & $0.8442(0.0065)$ & $0.8379(0.0086)$ & $0.8437(0.0105)$ & $0.8564(0.0118)$ & $0.8658(0.0127)$ \\
\hline
\end{tabular}




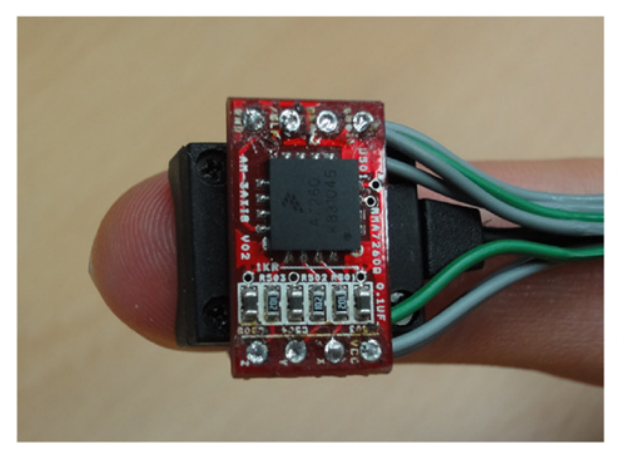

(a)

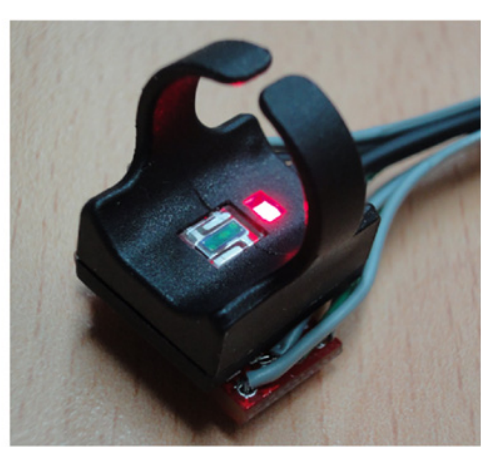

(b)

Figure 5. Reflected-type ring-shaped finger sensor used for the acquisition of the PPG signals. (a) Attached accelerometer to detect the motion artifacts and (b) LED and photo-detector of PPG sensing part.

Kalman smoother. PPG signals were measured with a commercial transmitted-type finger sensor (NONIN ${ }^{\circledR}$ finger clip 8000R) or a reflected-type ring-shaped finger sensor (LAXTHA ${ }^{\circledR}$ RP320), and the three-axis accelerometer (MMA7260Q) was attached to the finger sensor module to detect the movement of fingers (see figure 5). The data were acquired with a sampling rate of $128 \mathrm{~Hz}$ using our in-house potable device composed of a 12-bit $\mathrm{AD}$ converter, $32 \mathrm{MB}$ flash memory and USB connection with a computer. For the analog part of the developed device, the detected signal is processed by a high-pass filter with a $0.5 \mathrm{~Hz}$ cutoff at first, then amplified with a 25-time gain, and lastly processed using a low-pass filter with $10 \mathrm{~Hz}$ cutoff. Out of the three-dimensional data of the accelerometer, only the one-dimensional signal which was maximally correlated with the PPG signal was used for the adaptive removal of motion artifact. The PPG signals affected by motion artifacts were collected on the right index finger during finger or arm movement and the reference PPG signals were also measured on the left index finger which was in a fixed position.

Data were collected in motions, such as finger bending and arm swing, which are expected to take place during hospital monitoring or daily-life monitoring. To compare the performances of the algorithms, we collected PPG data during two well-defined motions to induce motion artifacts as in a real situation from four volunteers: finger bending and arm swing. We instructed each subject to bend their index fingers continuously with a frequency between 1 and $2 \mathrm{~Hz}$ along one direction and then swing their arms continuously with a frequency between 1 and $2 \mathrm{~Hz}$ along one direction, with their fingers inserted in the ring of PPG sensors. 20 trials of about $24 \mathrm{~s}$ durations were collected to obtain the measures such as mean and standard deviation for the purpose of the statistically meaningful comparison of the algorithms. All the PPG signals were acquired using a reflected-type ring-shaped sensor. Two examples of real PPG signals acquired during finger bending and arm swing for one subject are displayed in figure 6 in a similar manner to those of the simulation examples in figure 2 .

3.2.2. Results of real experiments. Four algorithms were applied to the each experimental data. All results are summarized in figure 7 and table 4 with eight different FIR filter tap orders $(8,16,24,32,40,48,56$ and 64). Eight examples of analysis on the representative trials for finger bending and arm swing belonging to each subject are arranged in table 3 , which gives the specific numerical values for the cross correlations between the reference signal and the signals of PPG with motion artifacts, and between the reference signal and reconstructed 

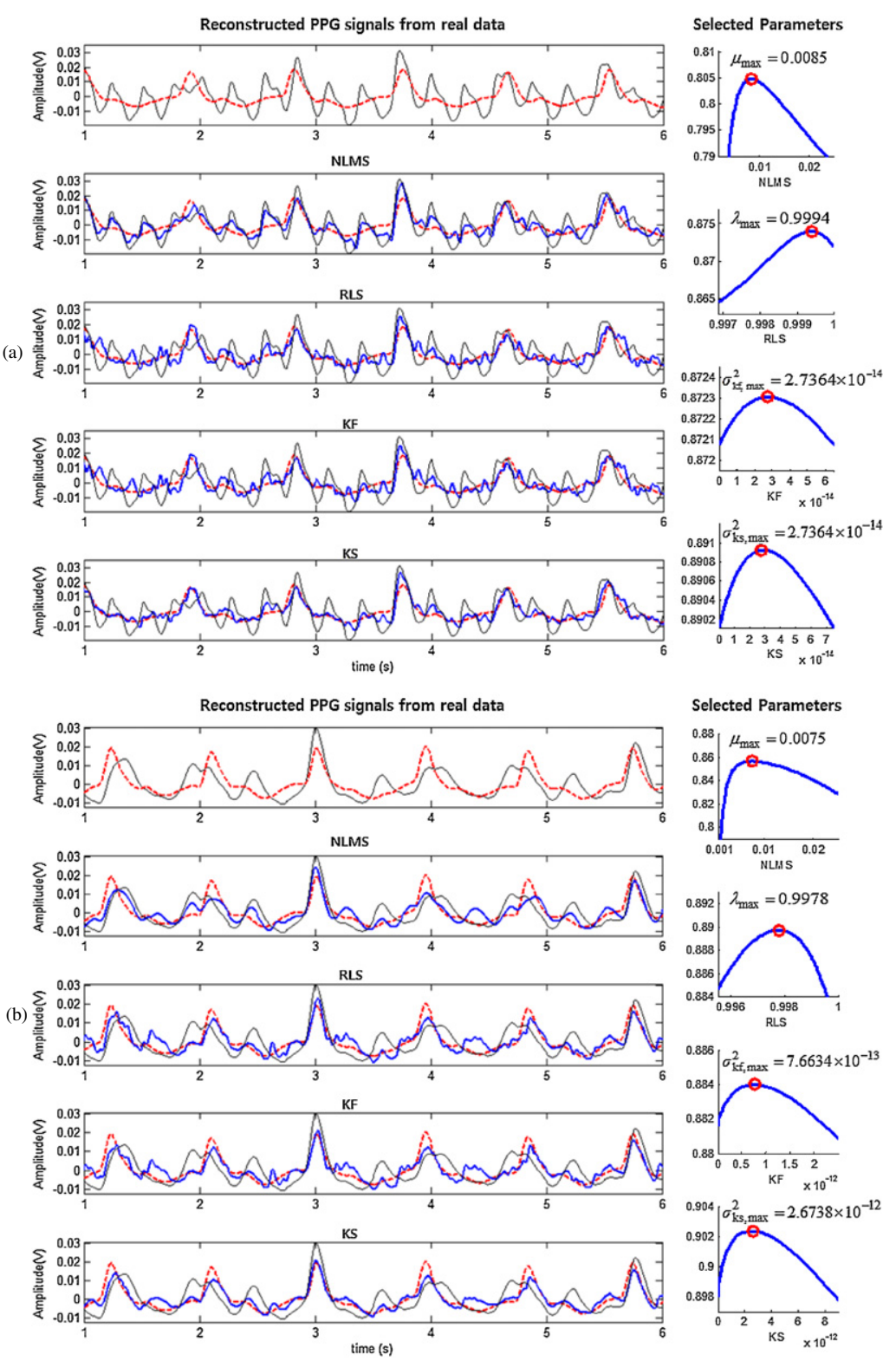

Figure 6. (a) One example of real experimental data (finger bending) of one subject is displayed. The adaptive FIR filter tap order is 16 in this sample analysis, and the results are displayed for $5 \mathrm{~s}$. The uppermost plot of the left column overlays the reference PPG signal (dotted line) and motion artifact-corrupted PPG signal (thin solid line) and the remaining lower plots represent the signals reconstructed by each algorithm (thick solid lines), i.e. NLMS, RLS, Kalman filter and fixedinterval Kalman smoother, which are overlaid over the reference and motion artifact-corrupted PPG signals. The cross correlation was 0.6458 between the reference PPG signal and the PPG with motion artifact before filtering. As in the plots of the right column, optimal parameters for each method are selected such that the reconstructed signals represent the maximal cross correlation with the reference PPG signal, respectively ( $x$-axis: parameter for each algorithm, $y$-axis: cross correlation). (b) Another example of arm swing of the same subject as (a) is displayed. The FIR filter tap order is 48 in this sample. The cross correlation was 0.6097 between the reference PPG signal and the PPG corrupted with motion artifact before filtering. Other explanations are the same as (a). 


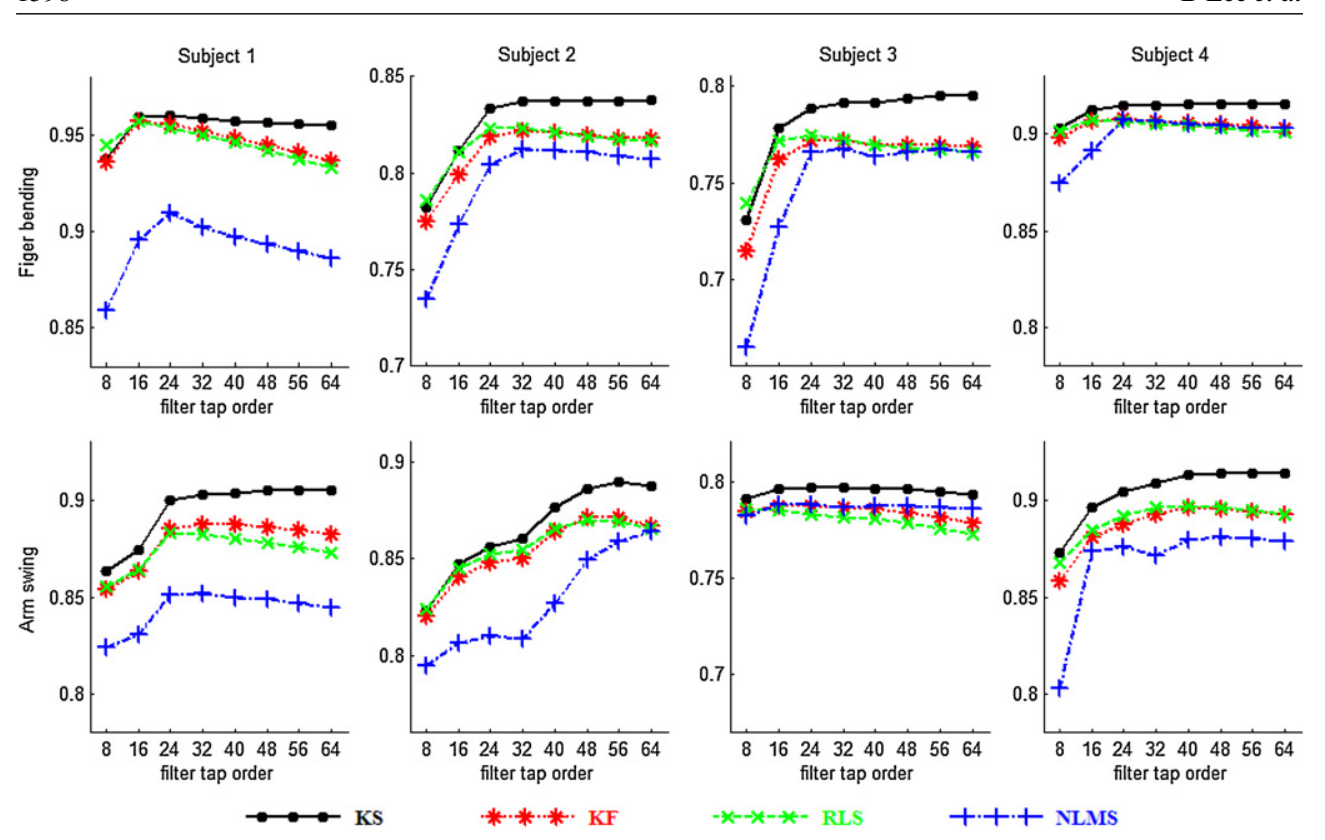

Figure 7. Mean values of cross correlations between the reference PPG signal and the filtered PPG signal for 20 trials of finger bending and arm swing obtained from four subjects. Mean cross correlations were plotted for all the combinations of datasets with eight different FIR filter tap orders $(8,16,24,32,40,48,56$ and 64). $\mathrm{KS}=$ fixed-interval Kalman smoother, $\mathrm{KF}=$ conventional Kalman filter, RLS = recursive least-squares method, NLMS = normalized least mean squares method. $Y$-axes represent the values of cross correlation between the filtered PPG signal and the reference PPG signal.

PPG signals acquired after processing using the four algorithms, respectively. In addition, table 3 shows the optimal values of parameters for the maximal cross correlation between the reconstructed PPG signals and the reference PPG signal ( $\mu_{\max }$ for NLMS, $\lambda_{\max }$ for RLS, $\sigma_{\mathrm{kf} \text {, max }}^{2}$ for the Kalman filter and $\sigma_{\mathrm{ks} \text {, max }}^{2}$ for the Kalman smoother). Unlike the analyses of the simulation data, the cross correlations were calculated between the signals with the first and last $2 \mathrm{~s}$ data omitted, to reduce the distorted portions. Figure 7 plots the mean values of the cross correlation between the reference PPG signal and the filtered PPG signal for 20 trials of finger bending and arm swing obtained from four subjects and table 4 shows the numerical values of the mean and standard deviation of cross correlations obtained using 20 trials of two motions for each subject.

For the analyses of all the experimental dataset with different filter tap orders, the fixedinterval Kalman smoother reconstructed PPG signals that were most correlated with the reference PPG signal on average in most situations. The results of the conventional Kalman filter and RLS were generally comparable to each other although in some cases there were slight differences between the results of the Kalman filter and those of the RLS filter. According to our experimental data, the NLMS method showed lower cross correlations than other algorithms generally. As the filter tap order increases, the cross correlations for each algorithm usually tended to increase and saturate above a certain limit of tap order which is 24 in most our data. In conclusion, the fixed-interval Kalman smoother produced the best outcomes out of the four algorithms in most combinations of the experimental PPG signals and filter tap orders especially after reaching the saturated values along the increasing tap order. 


\section{Discussion}

In this study, we implemented algorithms to eliminate the motion artifacts from the acquired PPG signals because motion artifacts distort PPG signals, making it impossible to extract accurate information of cardiovascular parameters, such as heart rate, HRV and oxygen saturation (Hayes and Smith 1998). Until now, many adaptive algorithms were used to remove motion artifacts from PPG signals. We propose an algorithm based on the fixed-interval Kalman smoother and we found that our proposed method generally showed superior performance to that of other adaptive filtering algorithms, like NLMS, RLS and the conventional Kalman filter, as evidenced by our analyses of simulation (figures 3 and 4, and table 2) and real experimental (figure 7 and tables 3 and 4) data. When considering the results on simulation data, we observed that the conventional Kalman filter and RLS algorithm usually show similar performances that were superior to that of the NLMS filter in most cases. However, the Kalman filter algorithm represents more stable estimates than RLS, since in some combination of simulation data with several filter tap orders, RLS reconstructed signals had much lower cross correlations with the original PPG signal than Kalman filtering (see figures 3 and 4). The real experimental data showed a similar trend to simulation data, despite showing more complexity than the simulation cases (figure 7). In the analyses of experimental data, the proposed method usually represents the superior results in comparison to the other algorithms by statistical sense. In addition, the Kalman filter and RLS filter generally show similar performances, which were superior to those of NLMS, although in some cases there were slight differences between the results of the Kalman filter and those of the RLS filter. In a few simulation and experimental cases, NLMS showed slight better performances than the Kalman and RLS algorithms (see figures 3 and 7). Interestingly, correlations of simulation results tended to decrease as the filter tap order increased. On the other hand, correlations of experimental data usually tended to increase as the filter tap order increased. This result may be because the simulation data are so simple that, if processed with many tap orders, they can be distorted more severely than when filtered with less order. However, since real data may represent a more complex situation than simulation data, more filter tap orders produce better results in most cases, which are saturated above a limit of order (roughly 24 in this study).

According to the previous studies, the RLS method and the Kalman filter showed comparable performances in other applications (Barnawi et al 2007, Chun et al 1998, Lanzi et al 2006). In addition, RLS can be described by a state-space model (Chun et al 1998) and it can be also recognized as a special case of Kalman filter (Sayed 2008). Lanzi et al (2006) reported that RLS represented better performances than NLMS from their results, which reflect the fact that RLS is usually more robust and converge faster than NLMS. Our study led to similar outcomes to previous reports. However, we found in some simulation cases that the Kalman filter produced better performances that the RLS method (the cases of 1 and $2 \mathrm{~Hz}$ MA with $-12.5,-10$ and $-7.5 \mathrm{dBs}$ in simulation 1 and all cases of 1 and $2 \mathrm{~Hz}$ MA in simulation 2 above the tap order of 24, see figures 3 and 4), and such situations are mainly the cases in which the frequencies $(1 \mathrm{~Hz}$ or $2 \mathrm{~Hz}$ ) of the original PPG were overlapped with those of motion artifact signals as well as added by another frequency component different from those of the original PPG. That is, the Kalman filter seems to produce more reliable results in complex and intractable situations. In this context, a study reported that the Kalman filter responded more quickly to the model changes in a simulation test than the RLS algorithm (Arnold et al 1998). However, this phenomenon did not appear prominently in the results of real experiment. Taken together, more tests and theoretical assessments will be necessary to identify definite evidences about the characteristics of these methods.

Traditional adaptive filtering methods, like NLMS, RLS and conventional Kalman filter, can be used for the real-time elimination of motion artifacts from PPG signals (on-line 
Table 3. Cross correlations between the reference PPG signal and the reconstructed PPG signals by four algorithms with regard to eight representative experimental data. $\mathrm{CC}_{\mathrm{MA}}$ is the cross correction between the reference PPG signal and the measured PPG distorted with real motion artifacts, $\mathrm{CC}_{\mathrm{KS}}$ is the cross correlation between the reference PPG signal and the PPG reconstructed by the fixed-interval Kalman smoother, $\mathrm{CC}_{\mathrm{KF}}$ is the cross correlation between the reference PPG signal and the PPG reconstructed by the conventional Kalman filter, $\mathrm{CC}_{\mathrm{RLS}}$ is the cross correlation between the reference PPG signal and the PPG reconstructed by the RLS filter, and $\mathrm{CC}_{\mathrm{NLMS}}$ is the cross correlation between the reference PPG signal and the PPG reconstructed by the NLMS filter. Values of optimal parameters for each algorithm are listed in the parentheses next to the values of cross correlation. MA $=$ motion artifact.

\begin{tabular}{|c|c|c|c|c|c|c|c|c|c|}
\hline \multirow[b]{2}{*}{ Dataset } & \multirow[b]{2}{*}{ Cross correlations } & \multicolumn{8}{|c|}{ Filter tap order } \\
\hline & & 8 & 16 & 24 & 32 & 40 & 48 & 56 & 64 \\
\hline \multirow[t]{4}{*}{ Subj 1 (Bending, $\mathrm{CC}_{\mathrm{MA}}=0.7196$ ) } & $\mathrm{CC}_{\mathrm{KS}}\left(\sigma^{2} \mathrm{ks}\right.$, max,$\left.\times 10^{-13}\right)$ & $0.9350(5.8052)$ & $0.9609(3.3313)$ & $0.9638(3.8275)$ & $0.9636(3.3313)$ & $0.9624(2.8994)$ & $0.9621(2.5235)$ & $0.9615(2.1964)$ & $0.9610(2.1964)$ \\
\hline & $\mathrm{CC}_{\mathrm{KF}}\left(\sigma_{\mathrm{kf}, \max }^{2} \times 10^{-14}\right)$ & $0.9299(88.049)$ & $0.9575(25.235)$ & 0.9591 (28.994) & $0.9561(8.3099)$ & $0.9524(7.2326)$ & $0.9481(4.7686)$ & $0.9470(1.1895)$ & $0.9430(2.381$ \\
\hline & $\mathrm{CC}_{\mathrm{RLS}}\left(\lambda_{\max }\right)$ & $0.9402(0.9947)$ & $0.9579(0.9984)$ & $0.9586(0.9992)$ & $0.9559(0.9994)$ & $0.9513(0.9999)$ & $0.9465(1)$ & $0.9452(1)$ & $0.9411(1)$ \\
\hline & $\operatorname{CC}_{\text {NLMS }}\left(\mu_{\max }\right)$ & $0.8488(0.0287)$ & $0.8447(0.0012)$ & $0.9242(0.0021)$ & $0.9255(0.0028)$ & $0.9237(0.0027)$ & $0.9206(0.0027)$ & $0.9176(0.0027)$ & $0.9138(0.0027)$ \\
\hline \multirow[t]{4}{*}{ Subj 1 (Swing, $\mathrm{CC}_{\mathrm{MA}}=0.7789$ ) } & $\mathrm{CC}_{\mathrm{KS}}\left(\sigma_{\mathrm{ks}, \max }^{2} \times 10^{-12}\right)$ & $0.8800(21.461)$ & $0.8929(14.150)$ & $0.9185(16.258)$ & $0.9214(14.150)$ & $0.9212(9.3293)$ & $0.9221(9.3293)$ & $0.9217(10.719)$ & $0.9192(16.258)$ \\
\hline & $\mathrm{CC}_{\mathrm{KF}}\left(\sigma^{2} \mathrm{kf}\right.$ max,$\left.\times 10^{-12}\right)$ & $0.8715(42.970)$ & $0.8816(28.331)$ & $0.9035(21.461)$ & $0.9031(16.258)$ & $0.9033(14.150)$ & $0.9059(14.150)$ & $0.9020(10.719)$ & $0.8883(9.3293)$ \\
\hline & $\mathrm{CC}_{\mathrm{RLS}}\left(\lambda_{\max }\right)$ & $0.8740(0.9950)$ & $0.8817(0.9952)$ & $0.8982(0.9962)$ & $0.8949(0.9967)$ & $0.8917(0.9969)$ & $0.8918(0.9970)$ & $0.8921(0.9973)$ & $0.8860(0.9974)$ \\
\hline & $\mathrm{CC}_{\mathrm{NLMS}}\left(\mu_{\max }\right)$ & $0.8710(0.0005)$ & $0.8751(0.0007)$ & $0.8728(0.0007)$ & $0.8715(0.0007)$ & $0.8761(0.0008)$ & $0.8784(0.0009)$ & $0.8780(0.0009)$ & $0.8770(0.0009)$ \\
\hline \multirow[t]{4}{*}{ Subj 2 (Bending, $\mathrm{CC}_{\mathrm{MA}}=0.5523$ ) } & $\mathrm{CC}_{\mathrm{KS}}\left(\sigma^{2} \mathrm{ks}\right.$ max,$\left.\times 10^{-13}\right)$ & 0.8728 (46.595) & $0.8804(30.721)$ & $0.9001(30.721)$ & 0.9009 (35.297) & $0.9002(20.255)$ & $0.8986(13.355)$ & $0.8976(11.623)$ & $0.8985(7.66$ \\
\hline & $\mathrm{CC}_{\mathrm{KF}}\left(\sigma^{2} \mathrm{kf}\right.$ max,$\left.\times 10^{-13}\right)$ & $0.8657(20.255)$ & $0.8705(8.8049)$ & $0.8876(7.6634)$ & 0.8889 (10.116) & $0.8887(5.8052)$ & $0.8865(3.8275)$ & $0.8806(4.3976)$ & $0.8818(4.397$ \\
\hline & $\mathrm{CC}_{\mathrm{RLS}}\left(\lambda_{\max }\right)$ & $0.8692(0.9960)$ & $0.8746(0.9963)$ & $0.8908(0.9969)$ & $0.8904(0.9979)$ & $0.8905(0.9982)$ & $0.8904(0.9980)$ & $0.8834(0.9983)$ & $0.8854(0.9985)$ \\
\hline & $\mathrm{CC}_{\mathrm{NLMS}}\left(\mu_{\max }\right)$ & $0.8452(0.0094)$ & $0.8525(0.0131)$ & $0.8702(0.0158)$ & $0.8822(0.0178)$ & $0.8765(0.0196)$ & $0.8764(0.0181)$ & $0.8734(0.0183)$ & $0.8699(0.0191)$ \\
\hline \multirow[t]{4}{*}{ Subj 2 (Swing, $\mathrm{CC}_{\mathrm{MA}}=0.7254$ ) } & $\mathrm{CC}_{\mathrm{KS}}\left(\sigma_{\mathrm{ks}, \max }^{2} \times 10^{-13}\right)$ & $0.8015(15.344)$ & $0.8246(11.623)$ & $0.8486(13.355)$ & 0.8547 (10.116) & $0.8548(8.8094)$ & $0.8652(7.6634)$ & 0.8737 (7.6634) & $0.8741(7.6634)$ \\
\hline & $\mathrm{CC}_{\mathrm{KF}}\left(\sigma_{\mathrm{kf}, \max }^{2} \times 10^{-13}\right)$ & 0.7954 (17.629) & 0.8169 (11.623) & $0.8358(10.116)$ & $0.8389(7.6634)$ & $0.8383(5.8052)$ & $0.8482(5.0526)$ & $0.8525(4.3976)$ & $0.8514(3.8275)$ \\
\hline & $\mathrm{CC}_{\mathrm{RLS}}\left(\lambda_{\max }\right)$ & $0.7952(0.9973)$ & $0.8191(0.9971)$ & $0.8375(0.9972)$ & $0.8392(0.9974)$ & $0.8364(0.9976)$ & $0.8417(0.9979)$ & $0.8390(0.9983)$ & $0.8370(0.9985)$ \\
\hline & $\mathrm{CC}_{\mathrm{NLMS}}\left(\mu_{\max }\right)$ & $0.7906(0.0029)$ & $0.8107(0.0045)$ & $0.8223(0.0054)$ & $0.8250(0.0058)$ & $0.8235(0.0062)$ & $0.8309(0.0067)$ & $0.8443(0.0074)$ & $0.8458(0.007$ \\
\hline \multirow[t]{4}{*}{ Subj 3 (Bending, $\mathrm{CC}_{\mathrm{MA}}=0.7378$ ) } & $\mathrm{CC}_{\mathrm{KS}}\left(\sigma^{2} \mathrm{ks}\right.$ max,$\left.\times 10^{-13}\right)$ & 0.8939 (13.355) & $0.9196(6.6699)$ & $0.9229(4.3976)$ & $0.9236(3.8275)$ & $0.9260(2.5235)$ & $0.9259(2.8994)$ & $0.9251(2.1964)$ & $0.9253(1.9116$ \\
\hline & 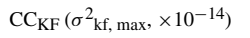 & 0.8824 (76.634) & $0.9109(9.5477)$ & $0.9151(\approx 0)$ & $0.9142(0.450)$ & $0.9139(1.8042)$ & $0.9127(2.0729)$ & $0.9130(\approx 0)$ & $0.9111(\approx 0)$ \\
\hline & $\mathrm{CC}_{\mathrm{RLS}}\left(\lambda_{\max }\right)$ & $0.8835(0.9978)$ & $0.9106(0.9994)$ & $0.9150(1)$ & $0.9141(1)$ & $0.9137(1)$ & $0.9125(1)$ & $0.9129(1)$ & $0.9109(1)$ \\
\hline & $\mathrm{CC}_{\text {NLMS }}\left(\mu_{\max }\right)$ & $0.8489(0.0046)$ & $0.9018(0.0056)$ & $0.9066(0.0055)$ & $0.9054(0.0064)$ & $0.9043(0.0064)$ & $0.9066(0.0066)$ & $0.9059(0.0071)$ & $0.9047(0.0075)$ \\
\hline \multirow[t]{4}{*}{ Subj 3 (Swing, $\mathrm{CC}_{\mathrm{MA}}=0.6648$ ) } & $\mathrm{CC}_{\mathrm{KS}}\left(\sigma_{\mathrm{ks}, \max }^{2} \times 10^{-13}\right)$ & 0.8895 (26.738) & 0.8975 (17.629) & $0.8994(15.344)$ & 0.8994 (10.116) & 0.9008 (6.6699) & $0.9000(5.8052)$ & $0.8991(5.0526)$ & $0.9009(4.3976)$ \\
\hline & $\mathrm{CC}_{\mathrm{KF}}\left(\sigma_{\mathrm{kff} \text { max }}^{2} \times 10^{-14}\right)$ & $0.8783(76.634)$ & $0.8845(33.3$ & & $0.8857(19.116)$ & & & & $0.8850(8.30$ \\
\hline & $\mathrm{CC}_{\mathrm{RLS}}\left(\lambda_{\max }\right)$ & $0.8784(0.9988)$ & $0.8822(0.9989)$ & $0.8806(0.9989)$ & $0.8809(0.9992)$ & $0.8839(0.9996)$ & $0.8828(0.9997)$ & $0.8821(0.9997)$ & $0.8804(0.999$ \\
\hline & $\mathrm{CC}_{\text {NLMS }}\left(\mu_{\max }\right)$ & $0.8739(0.0037)$ & $0.8805(0.0046)$ & $0.8782(0.0054)$ & $0.8762(0.0055)$ & $0.8801(0.0054)$ & $0.8838(0.0054)$ & $0.8833(0.0057)$ & $0.8816(0.0058)$ \\
\hline \multirow[t]{4}{*}{ Subj 4 (Bending, $\mathrm{CC}_{\mathrm{MA}}=0.7227$ ) } & $\mathrm{CC}_{\mathrm{KS}}\left(\sigma^{2} \mathrm{ks}\right.$ max,$\left.\times 10^{-12}\right)$ & $0.9079(6.1510)$ & $0.9178(4.0555)$ & $0.9236(4.0555)$ & $0.9231(2.3272)$ & $0.9229(1.7629)$ & $0.9230(1.3355)$ & $0.9232(1.1623)$ & $0.9236(1.0116)$ \\
\hline & $\mathrm{CC}_{\mathrm{KF}}\left(\sigma_{\mathrm{kf}, \max }^{2} \times 10^{-13}\right)$ & 0.9034 (26.738) & $0.9132(13.355)$ & $0.9163(15.344)$ & $0.9163(8.8049)$ & $0.9159(5.8052)$ & $0.9163(5.0526)$ & $0.9151(5.0526)$ & $0.9142(5.0526)$ \\
\hline & $\mathrm{CC}_{\mathrm{RLS}}\left(\lambda_{\max }\right)$ & $0.9120(0.9933)$ & $0.9177(0.9952)$ & $0.9156(0.9968)$ & $0.9140(0.9993)$ & $0.9136(0.9996)$ & $0.9136(0.9994)$ & $0.9120(0.9994)$ & $0.9105(0.99$ \\
\hline & $\mathrm{CC}_{\text {NLMS }}\left(\mu_{\max }\right)$ & $0.8822(0.0083)$ & $0.8983(0.0121)$ & $0.9164(0.0136)$ & $0.9146(0.0161)$ & $0.9135(0.0166)$ & $0.9113(0.0175)$ & $0.9105(0.0198)$ & $0.9101(0.02$ \\
\hline \multirow[t]{4}{*}{ Subj 4 (Swing, $\mathrm{CC}_{\mathrm{MA}}=0.5220$ ) } & $\mathrm{CC}_{\mathrm{KS}}\left(\sigma_{\mathrm{ks}, \max } \times 10^{-13}\right)$ & $0.9148(81.198)$ & $0.9318(30.721)$ & $0.9327(23.272)$ & $0.9351(17.629)$ & $0.9352(13.355)$ & $0.9355(10.116)$ & $0.9353(8.8049)$ & $0.9349(7.663$ \\
\hline & $\mathrm{CC}_{\mathrm{KF}}\left(\sigma_{\mathrm{kf}, \max }^{2} \times 10^{-13}\right)$ & $0.9070(26.738)$ & $0.9272(11.623)$ & $0.9268(6.6699)$ & $0.9283(5.0526)$ & $0.9274(5.0526)$ & $0.9259(4.3976)$ & $0.9253(3.8275)$ & $0.9251(2.89$ \\
\hline & $\mathrm{CC}_{\mathrm{RLS}}\left(\lambda_{\max }\right)$ & $0.9104(0.9969)$ & $0.9264(0.9984)$ & $0.9261(0.9989)$ & $0.9277(0.9991)$ & $0.9259(0.9991)$ & $0.9250(0.9991)$ & $0.9235(0.9992)$ & $0.9229(0.99$ \\
\hline & $\mathrm{CC}_{\mathrm{NLMS}}\left(\mu_{\max }\right)$ & $0.8708(0.0108)$ & $0.9164(0.0087)$ & $0.9154(0.0095)$ & $0.9182(0.0096)$ & $0.9205(0.0096)$ & $0.9191(0.0103)$ & $0.9162(0.0106)$ & $0.9151(0.0110$ \\
\hline
\end{tabular}


Table 4. Numerical values of mean and standard deviation for 20 trials of finger bending and arm swing motions measured from four subjects. Original values of mean and standard deviation for the cross correlations between the reference PPG signal and the raw PPG signals are also listed in the second column expressing motion types. Values of standard deviation are in the parentheses. $\mathrm{KS}=$ fixed-interval Kalman smoother, $\mathrm{KF}=$ conventional Kalman filter, $\mathrm{RLS}=$ recursive least-squares method, NLMS $=$ normalized least mean squares method

\begin{tabular}{|c|c|c|c|c|c|c|c|c|c|c|}
\hline \multirow[b]{2}{*}{ Subject } & \multirow{2}{*}{$\begin{array}{l}\text { Motion } \\
\text { type }\end{array}$} & \multirow{2}{*}{$\begin{array}{l}\text { Filter } \\
\text { type }\end{array}$} & \multicolumn{8}{|c|}{ FIR filter tap order } \\
\hline & & & 8 & 16 & 24 & 32 & 40 & 48 & 56 & 64 \\
\hline \multirow[t]{8}{*}{1} & Finger bending & KS & $0.9374(0.0137)$ & $0.9595(0.0089)$ & $0.9596(0.0080)$ & $0.9583(0.0083)$ & $0.9572(0.0088)$ & $0.9565(0.0093)$ & $0.9556(0.0097)$ & $0.9548(0.0098)$ \\
\hline & 0.7 & $\mathrm{KF}$ & $0.9359(0.0145)$ & $0.9572(0.0098)$ & $0.9553(0.0090)$ & $0.9516(0.0103)$ & $0.9482(0.0110)$ & $0.9447(0.0112)$ & $0.9405(0.0131)$ & $0.9362(0.0157)$ \\
\hline & & RLS & $0.9444(0.0138)$ & $0.9567(0.0090)$ & $0.9532(0.0088)$ & $0.9493(0.0103)$ & $0.9457(0.0112)$ & $0.9419(0.0112)$ & $0.9374(0.0126)$ & $0.9330(0.0148)$ \\
\hline & & NLMS & $0.8593(0.0362)$ & $0.8856(0.0613)$ & $0.9093(0.0679)$ & $0.9021(0.0713)$ & $0.8969(0.0724)$ & $0.8933(0.0724)$ & $0.8897(0.0739)$ & $0.8861(0.0740)$ \\
\hline & Arm swing & KS & $0.8637(0.0285)$ & $0.8745(0.0227)$ & $0.9002(0.0147)$ & $0.9036(0.0152)$ & $0.9042(0.0149)$ & $0.9054(0.0156)$ & $0.9054(0.0160)$ & $0.9051(0.0158)$ \\
\hline & $0.7567(0.0603)$ & $\mathrm{KF}$ & $0.8544(0.0302)$ & $0.8634(0.0254)$ & $0.8859(0.0166)$ & $0.8879(0.0168)$ & $0.8877(0.0170)$ & $0.8862(0.0182)$ & $0.8847(0.0180)$ & $0.8826(0.0183)$ \\
\hline & & RLS & $0.8559(0.0291)$ & $0.8643(0.0231)$ & $0.8835(0.0166)$ & $0.8828(0.0169)$ & $0.8808(0.0177)$ & $0.8788(0.0194)$ & $0.8764(0.0195)$ & $0.8735(0.0203)$ \\
\hline & & NLMS & $0.8243(0.0412)$ & $0.8310(0.0462)$ & $0.8513(0.0371)$ & $0.8524(0.0399)$ & $0.8502(0.0418)$ & $0.8492(0.0444)$ & $0.8471(0.0465)$ & $0.8445(0.0476)$ \\
\hline \multirow[t]{8}{*}{2} & Finger bending & KS & $0.7825(0.0914)$ & $0.8114(0.0825)$ & $0.8336(0.0718)$ & $0.8370(0.0709)$ & $0.8372(0.0711)$ & $0.8368(0.0709)$ & $0.8375(0.0704)$ & $0.8378(0.0704)$ \\
\hline & $0.5871(0.1317)$ & $\mathrm{KF}$ & $0.7752(0.0905)$ & $0.7990(0.0819)$ & $0.8190(0.0714)$ & $0.8215(0.0717)$ & $0.8209(0.0713)$ & $0.8194(0.0707)$ & $0.8183(0.0704)$ & $0.8179(0.0708)$ \\
\hline & & RLS & $0.7861(0.0843)$ & $0.8110(0.0743)$ & $0.8230(0.0711)$ & $0.8232(0.0729)$ & $0.8211(0.0740)$ & $0.8188(0.0741)$ & $0.8174(0.0738)$ & $0.8164(0.0749)$ \\
\hline & & NLMS & $0.7350(0.0930)$ & $0.7735(0.0929)$ & $0.8041(0.0726)$ & $0.8126(0.0706)$ & $0.8117(0.0703)$ & $0.8110(0.0693)$ & $0.8088(0.0701)$ & $0.8075(0.0700)$ \\
\hline & Arm swing & KS & & $0.8471(0.0527)$ & $0.8563(0.0530)$ & $0.8606(0.0515)$ & $0.8764(0.0480)$ & $0.8859(0.0377)$ & $0.0367)$ & $0.8875(0.0419)$ \\
\hline & $0.6212(0.1087)$ & $\mathrm{KF}$ & $0.8202(0.0599)$ & $0.8411(0.0573)$ & $0.8481(0.0593)$ & $0.8503(0.0592)$ & $0.8643(0.0569)$ & $0.8715(0.0493)$ & $0.8719(0.0516)$ & $0.8675(0.0584)$ \\
\hline & & RLS & & 0544) & & $0.8546(0.0572)$ & $0.8658(0.0560)$ & $0.8705(0.0490)$ & 0.8697 & 0.8658( \\
\hline & & NLMS & $0.7951(0.0810)$ & $0.8066(0.0787)$ & $0.8103(0.0806)$ & $0.8088(0.0835)$ & $0.8273(0.0769)$ & $0.8496(0.0615)$ & $0.8592(0.0458)$ & $0.8644(0.0453)$ \\
\hline \multirow[t]{8}{*}{3} & Finger bending & KS & $0.7307(0.1768)$ & $0.7779(0.1550)$ & $0.7887(0.1472)$ & $0.7913(0.1444)$ & $0.7912(0.1442)$ & $0.7939(0.1430)$ & $0.7952(0.1409)$ & $0.7953(0.1403)$ \\
\hline & $0.5885(0.1885)$ & $\mathrm{KF}$ & $149(0$. & $0.7619(0.1546)$ & $0.7714(0.1473)$ & $0.7717(0.1455)$ & $0.7694(0.1446)$ & $0.7698(0.1428)$ & $0.7699(0.1410)$ & $0.7689(0.1404)$ \\
\hline & & RLS & $0.7395(0.1610)$ & $0.7720(0.1486)$ & $0.7750(0.1453)$ & $0.7727(0.1449)$ & $0.7694(0.1444)$ & $0.7684(0.1436)$ & $0.7671(0.1424)$ & $0.7661(0.1414)$ \\
\hline & & NLM & $0.6650(0.1966)$ & $0.7274(0.1716)$ & $0.7660(0.1486)$ & $0.7676(0.1454)$ & $0.7634(0.1463)$ & $0.7658(0.1445)$ & $0.7676(0.1419)$ & $0.7660(0.1417)$ \\
\hline & Arm swing & KS & $0.7908(0.1355)$ & $0.7962(0.1362)$ & $0.7971(0.1375)$ & $0.7966(0.1384)$ & $0.7965(0.1378)$ & $0.7959(0.1369)$ & $0.7945(0.1373)$ & $0.7933(0.1383)$ \\
\hline & $0.7016(0.1234)$ & $\mathrm{KF}$ & $0.7847(0.1400)$ & $0.7876(0.1421)$ & & $0.7867(0.1435)$ & & & & $0.7789(0.1433)$ \\
\hline & & RLS & $0.7858(0.1405)$ & $0.7856(0.1423)$ & $0.7833(0.1423)$ & $0.7819(0.1419)$ & $0.7809(0.1412)$ & $0.7787(0.1413)$ & $0.7759(0.1417)$ & $0.7731(0.1417)$ \\
\hline & & NLM & 0.7 & & & $0.7870(0.1412)$ & 0.7871( & & 0.7868 & $0.7863(0.1409)$ \\
\hline \multirow[t]{8}{*}{4} & Finger bending & KS & $034(0.0487)$ & $0.9130(0.0437)$ & $0.9153(0.0427)$ & $0.9152(0.0428)$ & $0.9154(0.0426)$ & $0.9155(0.0424)$ & $0.9156(0.0421)$ & $0.9155(0.0422)$ \\
\hline & $0.7539(0.0906)$ & $\mathrm{KF}$ & $978(0.0490)$ & $0.9070(0.0446)$ & $0.9083(0.0434)$ & $0.9072(0.0442)$ & $0.9064(0.0436)$ & $0.9056(0.0427)$ & $0.9047(0.0424)$ & $0.9034(0.0432)$ \\
\hline & & RLS & $0.9021(0.0470)$ & $0.9079(0.0443)$ & $0.9066(0.0452)$ & $0.9058(0.0454)$ & $0.9048(0.0447)$ & $0.9034(0.0439)$ & $0.9020(0.0435)$ & $0.9007(0.0439)$ \\
\hline & & NLMS & $0.8748(0.0601)$ & $0.8913(0.0543)$ & $0.9080(0.0462)$ & $0.9071(0.0458)$ & $0.9057(0.0458)$ & $0.9048(0.0461)$ & $0.9036(0.0463)$ & $0.9030(0.0464)$ \\
\hline & Arm swil & KS & & $0.8970(0.0227)$ & $0.9050(0.0208)$ & $0.9092(0.0177)$ & $0.9136(0.0160)$ & $0.9143(0.0159)$ & $0.9143(0.0164)$ & $0.9145(0.0163)$ \\
\hline & $0.5125(0.0725)$ & $\mathrm{KF}$ & $0.8590(0.0332)$ & $0.8814(0.0280)$ & $0.8881(0.0247)$ & $0.8930(0.0232)$ & $0.8963(0.0217)$ & $0.8960(0.0232)$ & $0.8943(0.0244)$ & $0.8931(0.0246)$ \\
\hline & & RLS & & & $0.8921(0.0222)$ & $0.8964(0.0212)$ & & $0.8970(0.0215)$ & $0.8950(0.0226)$ & $0.8930(0.0235)$ \\
\hline & & NLMS & $0.8032(0.0346)$ & $0.8738(0.0258)$ & $0.8760(0.0252)$ & $0.8719(0.0256)$ & $0.8800(0.0261)$ & $0.8816(0.0267)$ & $0.8804(0.0271)$ & $0.8790(0.0276)$ \\
\hline
\end{tabular}


processing). Out of these methods, the method based on the Kalman formula usually gave more stable and accurate results from the present study. The fixed-interval Kalman smoother mostly showed the best performances among the algorithms analyzed, but this method can be used only after data acquisition is complete (off-line processing). If the fixed-lag Kalman smoother is used in motion artifact elimination, the real-time processing will be possible in spite of the considerable computational cost (Simon 2006, Vauhkonen et al 2001). Therefore, our proposed method and other real-time algorithms can be used in a complementary way. To the limit of the present study, our proposed method based on the fixed-interval Kalman smoother generally showed superior performance to other algorithms, but additional studies will be necessary to guarantee its proper evaluation. In future work, we will investigate using two or more directional information of an accelerometer and more effective algorithms with less computational burden.

\section{Acknowledgments}

This study was supported by the IT R\&D program of MKE/KEIT (2009-S-014-01, On the development of Sensing-Based Emotive Service Mobile Handheld Devices) and also by a grant from the Institute of Medical System Engineering in the GIST, Korea.

\section{References}

Arnold M, Miltner W H R, Witte H, Bauer R and Braun C 1998 Adaptive AR modeling of nonstationary time series by means of Kalman filtering IEEE Trans. Biomed. Eng. 45 553-62

Asada H H, Shaltis P, Reisner A, Rhee S and Hutchingson R C 2003 Mobile monitoring with wearable photoplethysmographic biosensors IEEE Eng. Med. Biol. Mag. 22 28-40

Baek H J, Kim K K, Kim J S, Lee B and Park K S 2010 Enhancing the estimation of blood pressure using pulse arrival time and two confounding factors Physiol. Meas. 31 145-57

Barnawi A, Albakkar A and Malik O P 2007 RLS and Kalman filter identifiers based adaptive SVC controller 39th North American Power Symp. 2007 (NAPS '07) pp 615-22

Bonmassar G, Purdon P L, Jääskeläinen I P, Chiappa K, Solo V, Brown E N and Belliveau J W 2002 Motion and ballistocardiogram artifact removal for interleaved recording of EEG and EPs during MRI Neuroimage 16 1127-41

Chun B, Kim B and Lee Y H 1998 Generalization of exponentially weighted RLS algorithm based on a state-space model ISCAS '98-Proc. of the 1998 IEEE Int. Symp. on Circuits and Systems pp 198-201

Foo J Y A 2006 Comparison of wavelet transformation and adaptive filtering in restoring artefact-induced time-related measurement Biomed. Signal Process. Control $193-8$

Georgiadis S D, Ranta-aho P O, Tarvainen M P and Karjalainen P A 2005 Single-trial dynamical estimation of event-related potentials: a Kalman filter-based approach IEEE Trans. Biomed. Eng. 52 1397-406

Gibbs P T, Wood L B and Asada H H 2005 Active motion artifact cancellation for wearable health monitoring sensors using collocated MEMS accelerometers Smart Structures and Materials 2005: Sensors and Smart Structures Technologies for Civil, Mechanical, and Aerospace Systems International Society for Optical Engineering (San Diego, CA, USA) pp 811-9

Grewal M S and Andrews A P 2008 Kalman Filtering: Theory and Practice Using MATLAB (Hoboken, NJ: Wiley)

Han H, Kim M-J and Kim J 2007 Development of real-time motion artifact reduction algorithm for a wearable photoplethysmography 29th Апnи. Int. Conf. of the IEEE EMBS: Cité Internationale (Lyon, France) pp 1538-41

Hayes M H 1996 Statistical Digital Signal Processing and Modeling (New York: Wiley)

Hayes M J and Smith P R 1998 Artifact reduction in photoplethysmography Appl. Opt. 37 7437-46

Haykin S 2002 Adaptive Filter Theory (Englewood Cliffs, NJ: Prentice Hall)

In M H, Lee S Y, Park T S, Kim T-S, Cho M H and Ahn Y B 2006 Ballistocardiogram artifact removal from EEG signals using adaptive filtering of EOG signals Physiol. Meas. 27 1227-40

Kim B S and Yoo S K 2006 Motion artifact reduction in photoplethysmography using independent component analysis IEEE Trans. Biomed. Eng. $\mathbf{5 3} 566-8$ 
Kunesch E, Binkofski F and Freund H-J 1989 Invariant temporal characteristics of manipulative hand movements Exp. Brain Res. 78 539-46

Lanzi P L, Loiacono D, Wilson S W and Goldberg D E 2006 Prediction update algorithms for XCSF: RLS, Kalman filter, and gain adaptation Genetic and Evolutionary Computation Conf. (Seattle, Washington, USA) pp 1505-12

Lu S, Zhao H, Ju K, Shin K, Lee M, Shelley K and Chon K H 2008 Can photoplethysmography variability serve as an alternative approach to obtain heart rate variability information? J. Clin. Monit. Comput. 22 23-9

Sayed A H 2008 Kalman filtering and RLS Adaptive Filters (Hoboken, NJ: Wiley) pp 501-14 chapter 31

Sayed A H and Kailath T 1994 A state-space approach to adaptive RLS filtering IEEE Signal Process. Mag. 11 18-60

Seyedtabaii S and Seyedtabaii L 2008 Kalman filter based adaptive reduction of motion artifact from photoplethysmographic signal World Acad. Sci. Eng. Technol. 37 173-6

Shelley K H 2007 Photoplethysmography: beyond the calculation of arterial oxygen saturation and heart rate Anesth. Analg. 105 S31-6

Simon D 2006 Optimal State Estimation: Kamlan, Hळ, and Nonlinear Approaches (Hoboken, NJ: Wiley)

Tarvainen M P, Georgiadis S D, Ranta-aho P O and Karjalainen P A 2006 Time-varying analysis of heart rate variability signals with a Kalman smoother algorithm Physiol. Meas. 27 225-39

Tarvainen M P, Hiltunen J K, Ranta-aho P O and Karjalainen P A 2004 Estimation of nonstationary EEG with Kalman smoother approach: an application to event-related synchronization (ERS) IEEE Trans. Biomed. Eng. 51 516-24

Vauhkonen P J, Vauhkonen M and Kaipio J P 2001 Fixed-lag smoothing and state estimation in dynamic electrical impedence tomography Int. J. Numer. Methods Eng. 50 2195-209

Xiong Y and Quek F 2006 Hand motion gesture frequency properties and multimodal discourse analysis Int. J. Comput. Vis. $69353-71$ 